\title{
Transgressive loop group extensions
}

\author{
Konrad Waldorf
}

\begin{abstract}
A central extension of the loop group of a Lie group is called transgressive, if it corresponds under transgression to a degree four class in the cohomology of the classifying space of the Lie group. Transgressive loop group extensions are those that can be explored by finite-dimensional, higher-categorical geometry over the Lie group. We show how transgressive central extensions can be characterized in a loop-group theoretical way, in terms of loop fusion and thin homotopy equivariance.
\end{abstract}

\section{Contents}

1 Introduction 1 直

2 Fusion and thin homotopy equivariance over loop groups

3 Features of fusion and thin homotopy equivariance 10

3.1 Flat loops and retraction . . . . . . . . . . . . . . . . . . . 10

3.2 Loop concatenation . . . . . . . . . . . . . . . . . . . . . 14

3.3 Disjoint-commutativity . . . . . . . . . . . . . . . . . . . . . . . . .

4 Integrable thin homotopy equivariant structures

5 Transgression-regression machine 18

5.1 Multiplicative bundle gerbes . . . . . . . . . . . . . . . . . . . . . . . 18

5.2 Transgressive central extensions . . . . . . . . . . . . . . . . . . . . . 27

5.3 Regression and equivalence result . . . . . . . . . . . . . . . . . . . . 24

6 Segal-Witten reciprocity 27

A Regression of trivial fusion bundles

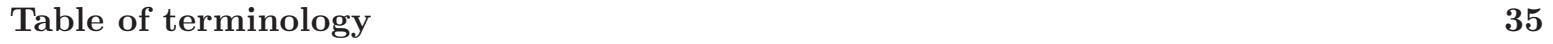

References 30

\section{Introduction}

The present article is about a Lie group $G$, its loop group $L G:=C^{\infty}\left(S^{1}, G\right)$ and central extensions

$$
1 \rightarrow \mathrm{U}(1) \rightarrow \mathcal{L} \rightarrow L G \rightarrow 1
$$

in the category of Fréchet Lie groups. Some central extensions $\mathcal{L}$ can be obtained from structure over $G$ called multiplicative bundle gerbe with connection via a procedure called transgression. These central 
extensions are called transgressive. In the case of $G$ compact, transgression induces a map

$$
\mathrm{H}^{4}(B G, \mathbb{Z}) \rightarrow\left\{\begin{array}{c}
\text { Isomorphism classes of } \\
\text { central extensions of } L G
\end{array}\right\}
$$

and a central extension is transgressive if and only if it is in the image of that map. An example of a transgressive central extension is the universal central extension of the loop group of a compact simply-connected Lie group $G$ : it corresponds to a generator of $\mathrm{H}^{4}(B G, \mathbb{Z}) \cong \mathbb{Z}$.

The goal of this article is to characterize transgressive central extensions for arbitrary connected Lie groups in purely loop group-theoretic terms. For this purpose we consider two relations on $L G$ :

1. thin homotopy: a homotopy between two loops is called thin, if its differential has nowhere full rank; these are homotopies that sweep out a surface of zero area.

2. loop fusion: it relates two loops that share a common line segment to a new loop with that segment deleted.

We introduce the notion of a thin fusion extension: a central extension

$$
1 \rightarrow \mathrm{U}(1) \rightarrow \mathcal{L} \rightarrow L G \rightarrow 1
$$

in which both relations are lifted in a consistent way to $\mathcal{L}$. Thin fusion extensions form a subclass of central extensions of $L G$ with several interesting properties. For example, we show that they are disjoint-commutative: if $p_{1}, p_{2} \in \mathcal{L}$ project to loops supported on disjoint subintervals of $S^{1}$, then they commute, $p_{1} \cdot p_{2}=p_{2} \cdot p_{1}$.

The main results of this paper are summarized in the following main theorem.

Theorem A. Let $G$ be a connected Lie group. A central extension $\mathcal{L}$ of $L G$ is transgressive if and only if it can be equipped with the structure of a thin fusion extension. Moreover, transgression is a group isomorphism

$$
\left\{\begin{array}{c}
\text { Isomorphism classes of } \\
\text { multiplicative bundle gerbes } \\
\text { over } G \text { that admit connections }
\end{array}\right\} \cong\left\{\begin{array}{c}
\text { Isomorphism classes } \\
\text { of thin fusion } \\
\text { extensions of } L G
\end{array}\right\} \text {. }
$$

If $G$ is compact, both groups are isomorphic to $\mathrm{H}^{4}(B G, \mathbb{Z})$.

As a consequence of Theorem $\mathrm{A}$, we obtain that transgressive central extensions are disjoint-commutative; this provides an accessible necessary condition for the transgressivity of a central extension.

The present work is a contribution to the programme of exploring the geometry of loop groups via finite-dimensional, higher geometry over Lie groups. Our main theorem determines the class of central extensions of loop groups that are accessible by such methods: thin fusion extensions.

Transgression of gerbes has first been defined by Gawȩdzki in relation with two-dimensional conformal field theories Gaw88, and then by Brylinski and McLaughlin in the setting of sheaves of groupoids Bry93, BM94. The multiplicative bundle gerbes we use here have been introduced by Carey et al. in $\left.\mathrm{CJM}^{+} 05\right]$, and transgression of those has been developed in [Wal10].

The question which central extensions are transgressive has been studied before by Brylinski and McLaughlin [BM94, BM96]. For connected semisimple complex Lie groups, they obtained a characterization in terms of the so-called Segal-Witten reciprocity law. In [BM94] it is incorrectly stated Bry 
that this reciprocity law also holds for non-complex Lie groups. Indeed, we provide a counterexample to that statement and prove that only a weaker version of the reciprocity law holds for transgressive central extensions of general Lie groups. We also provide an example of a central extension that is not transgressive and yet satisfies this weaker version of the reciprocity law. We come to the conclusion that no version of the reciprocity law appropriately characterizes transgressive central extensions of general Lie groups. It was the main motivation for writing this article to attack the open characterization problem from a different angle, namely via fusion and thin homotopy equivariance.

The results of this article are based on previous work on transgression Wal12b, Walb, Wal12c. A summary of these three papers on only three pages can be found in Wal12c, Section 1]. The main result is that transgression for general smooth manifolds $X$ establishes an equivalence between various categories of gerbes over $X$ and corresponding categories of $S^{1}$-bundles over the free loop space $L X$, equipped with structure rendering them compatible with fusion and thin homotopy. The present paper can be seen as an extension of these results to a multiplicative setting.

The organization of the present paper is as follows. In Section 2 we introduce the basic definitions of fusion and thin homotopy equivariance in loop group extensions. In Section 3 we provide a list of features that follow from the presence of these structures, among them disjoint-commutativity (Theorem 3.3.1). In Section 4 we formulate an integrability condition for thin homotopy equivariant structures on which our notion of thin fusion extensions is based (Definition 4.7). In Section 5 we discuss multiplicative bundle gerbes and their transgression, and introduce our definition of transgressive central extensions (Definition 5.2.1). Then we give a proof of Theorem $\mathrm{A}$ (split into Proposition 5.2.2 and Corollaries 5.3.2 5.3.4). In Section 6 we prove our weaker version of the Segal-Witten reciprocity law (Theorem 6.3) and provide the two examples that indicate the above-mentioned problems (Examples 6.6 and 6.7).

Throughout the paper, we continuously look at two classes of examples: an explicit model of the universal central extension of the loop group of a compact simply-connected Lie group, and various central extensions of $L \mathrm{U}(1)$, of which some turn out to be transgressive and others not. For the convenience of the reader we include on Page 35 a table summarizing some terminology we use in this paper.

Acknowledgements. This work is supported by the DFG network "String Geometry" (project code 594335).

\section{Fusion and thin homotopy equivariance over loop groups}

In this section $G$ is a Lie group and

$$
1 \rightarrow \mathrm{U}(1) \rightarrow \mathcal{L} \rightarrow L G \rightarrow 1
$$

is a central extension of Fréchet Lie groups [PS86]. We introduce structures on $\mathcal{L}$ that lift loop fusion and thin homotopy, and discuss the interplay between them.

By $P G$ we denote the set of paths $\gamma:[0,1] \rightarrow G$ with sitting instants, i.e. they are constant near the endpoints. $P G$ is not a Fréchet manifold, but can be treated as a diffeological space. Instead of charts, a diffeological space $X$ has plots: maps $c: U \rightarrow X$ defined on open subsets $U \subseteq \mathbb{R}^{n}$, for all $n \in \mathbb{N}$, satisfying three natural axioms, see e.g. [Z13. In case of $P G$, a map $c: U \rightarrow P G$ is a plot 
if and only if $U \times[0,1] \longrightarrow G:(x, t) \mapsto c(x)(t)$ is smooth 1

We remark that Fréchet manifolds embed fully faithfully into diffeological spaces [Los92. Thus, every Fréchet manifold can be regarded as a diffeological space (the plots are are just all smooth maps), and a map between two Fréchet manifolds is smooth in the Fréchet sense if and only if it is smooth in the diffeological sense.

We denote by $P G^{[k]}$ the $k$-fold fibre product of $P G$ over the evaluation map

$$
\text { ev }: P G \longrightarrow G \times G: \gamma \longmapsto(\gamma(0), \gamma(1)),
$$

i.e. $P G^{[k]}$ consists of $k$-tuples of paths all sharing a common initial point and a common end point. Due to the sitting instants, we have a well-defined smooth map

$$
\cup: P G^{[2]} \rightarrow L G:\left(\gamma_{1}, \gamma_{2}\right) \longmapsto \overline{\gamma_{2}} \star \gamma_{1},
$$

where $\star$ denotes the path concatenation, and $\bar{\gamma}$ denotes the reversed path. The set $P G^{[3]}$ is the modelling space for loop fusion: if $\left(\gamma_{1}, \gamma_{2}, \gamma_{3}\right) \in P G^{[3]}$, then we have the two loops $\tau_{12}:=\gamma_{1} \cup \gamma_{2}$ and $\tau_{23}:=\gamma_{2} \cup \gamma_{3}$ which have the common segment $\gamma_{2}$. Its deletion gives the new loop $\tau_{13}:=\gamma_{1} \cup \gamma_{3}$. Loop fusion is multiplicative and strictly associative.

\section{Definition 2.1.}

(a) A fusion product on $\mathcal{L}$ is a smooth bundle morphism

$$
\lambda: \operatorname{pr}_{12}^{*} \cup^{*} \mathcal{L} \otimes \operatorname{pr}_{23}^{*} \cup^{*} \mathcal{L} \longrightarrow \operatorname{pr}_{13}^{*} \cup^{*} \mathcal{L}
$$

over $P G^{[3]}$ that is associative in the sense that

$$
\lambda\left(\lambda\left(p_{12} \otimes p_{23}\right) \otimes p_{34}\right)=\lambda\left(p_{12} \otimes \lambda\left(p_{23} \otimes p_{34}\right)\right)
$$

for all $p_{i j} \in \mathcal{L}_{\gamma_{i} \cup \gamma_{j}}$ and all $\left(\gamma_{1}, \gamma_{2}, \gamma_{3}, \gamma_{4}\right) \in P G^{[4]}$.

(b) A fusion product $\lambda$ is called multiplicative if

$$
\lambda\left(p_{12} \otimes p_{23}\right) \cdot \lambda\left(p_{12}^{\prime} \otimes p_{23}^{\prime}\right)=\lambda\left(p_{12} p_{12}^{\prime} \otimes p_{23} p_{23}^{\prime}\right)
$$

for all elements $p_{i j} \in \mathcal{L}_{\gamma_{i} \cup \gamma_{j}}, p_{i j}^{\prime} \in \mathcal{L}_{\gamma_{i} \cup \gamma_{j}^{\prime}}$ and all $\left(\gamma_{1}, \gamma_{2}, \gamma_{3}\right),\left(\gamma_{1}^{\prime}, \gamma_{2}^{\prime}, \gamma_{3}^{\prime}\right) \in P G^{[3]}$.

Here $\operatorname{pr}_{i j}: P G^{[3]} \rightarrow P G^{[2]}$ is the projection to the indexed factors, and $\mathcal{L}_{\tau}$ denotes the fibre of $\mathcal{L}$ over a loop $\tau \in L G$. If $\mathcal{L}^{\prime}$ is another central extension equipped with a fusion product $\lambda^{\prime}$, then an isomorphism $\varphi: \mathcal{L} \longrightarrow \mathcal{L}^{\prime}$ is called fusion-preserving, if $\varphi\left(\lambda\left(p_{12} \otimes p_{23}\right)\right)=\lambda^{\prime}\left(\varphi\left(p_{12}\right) \otimes \varphi\left(p_{23}\right)\right)$ for all elements $p_{i j} \in \mathcal{L}_{\gamma_{i} \cup \gamma_{j}}$ and all $\left(\gamma_{1}, \gamma_{2}, \gamma_{3}\right) \in P G^{[3]}$.

A homotopy between loops is the same thing as a path in the loop space: if $\gamma:[0,1] \longrightarrow L G$ is a path, then

$$
h_{\gamma}:[0,1] \times S^{1} \longrightarrow G:(t, z) \mapsto \gamma(t)(z)
$$

is the corresponding homotopy. The rank of $h$ can at most be two. If it is less than two we call the path $\gamma$ and the homotopy $h_{\gamma}$ thin. A path $\gamma$ is thin if and only if $h_{\gamma}^{*} \omega=0$ for all 2-forms $\omega \in \Omega^{2}(G)$, this leads to the saying that thin homotopies "sweep out a surface of zero area".

\footnotetext{
${ }^{1} \mathrm{~A}$ referee suggested to use paths all of whose higher derivatives vanish at the end-points, as opposed to sitting instants, as this would simplify some of the arguments in Sections 3.2 and 3.3 In order to stay consistent with my other papers Wal12b Walb Wal12c , on which Sections 4 and 5 rely on, I have decided to stick to sitting instants.
} 
We denote by $L G_{\text {thin }}^{2} \subseteq L G \times L G$ the diffeological space consisting of pairs $\left(\tau_{1}, \tau_{2}\right)$ of thin homotopic loops, i.e. there exists a homotopy $h:[0,1] \times S^{1} \rightarrow G$ of rank one. The plots are smooth maps $c: U \rightarrow L G_{\text {thin }}^{2}$ such that locally the thin homotopies can be chosen in smooth families, see Wal12c, Section 3.1].

\section{Definition 2.2 .}

(a) A thin homotopy equivariant structure on $\mathcal{L}$ is a smooth bundle isomorphism

$$
d: \operatorname{pr}_{1}^{*} \mathcal{L} \longrightarrow \operatorname{pr}_{2}^{*} \mathcal{L}
$$

over $L G_{\text {thin }}^{2}$ that satisfies the cocycle condition $d_{\tau_{2}, \tau_{3}} \circ d_{\tau_{1}, \tau_{2}}=d_{\tau_{1}, \tau_{3}}$ for any triple $\left(\tau_{1}, \tau_{2}, \tau_{3}\right)$ of thin homotopic loops.

(b) A thin homotopy equivariant structure $d$ is called multiplicative if

$$
d_{\tau_{0} \gamma_{0}, \tau_{1} \gamma_{1}}(p \cdot q)=d_{\tau_{0}, \tau_{1}}(p) \cdot d_{\gamma_{0}, \gamma_{1}}(q)
$$

for all $\left(\left(\tau_{0}, \gamma_{0}\right),\left(\tau_{1}, \gamma_{1}\right)\right) \in L(G \times G)_{\text {thin }}^{2}$ and all $p \in \mathcal{L}_{\tau_{0}}, q \in \mathcal{L}_{\gamma_{0}}$.

Note that $\left(\left(\tau_{0}, \gamma_{0}\right),\left(\tau_{1}, \gamma_{1}\right)\right) \in L(G \times G)_{\text {thin }}^{2}$ means that there exists a thin path $(\tau, \gamma)$ in $L(G \times G)$ connecting $\left(\tau_{0}, \gamma_{0}\right)$ with $\left(\tau_{1}, \gamma_{1}\right)$. It is necessary, but not sufficient, that the paths $\tau, \gamma$, and $\tau \gamma$ in $L G$ are separately thin.

If $\mathcal{L}^{\prime}$ is another central extension equipped with a thin homotopy equivariant structure $d^{\prime}$, then an isomorphism $\varphi: \mathcal{L} \longrightarrow \mathcal{L}^{\prime}$ is called thin if $\varphi\left(d_{\tau_{0}, \tau_{1}}(p)\right)=d_{\tau_{0}, \tau_{1}}^{\prime}(\varphi(p))$.

A thin homotopy equivariant structure $d$ induces an equivariant structure on $\mathcal{L}$ for the action of the group $\operatorname{Diff}^{+}\left(S^{1}\right)$ of orientation-preserving diffeomorphisms of the circle on $L G$ by precomposition. Indeed, suppose $\varphi$ is an orientation-preserving diffeomorphism, $\tau \in L G$ and $p \in \mathcal{L}_{\tau}$. Since $\operatorname{Diff}^{+}\left(S^{1}\right)$ is connected, there exists a path $\varphi_{t} \in \operatorname{Diff}^{+}\left(S^{1}\right)$ with $\varphi_{0}=\operatorname{id}_{S^{1}}$ and $\varphi_{1}=\varphi$. The map $\gamma:[0,1] \longrightarrow L G: t \longmapsto \tau \circ \varphi_{t}$ is a thin path; in particular, $\tau$ and $\tau \circ \varphi$ are thin homotopic. We define

$$
p \cdot \varphi:=d_{\tau, \tau \circ \varphi}(p) \in \mathcal{L}_{\tau \circ \varphi} .
$$

Lemma 2.3. Formula (2.1) defines a smooth action of $\operatorname{Diff}^{+}\left(S^{1}\right)$ on $\mathcal{L}$. If $d$ is multiplicative, it is an action by group homomorphisms and acts trivially on the central $\mathrm{U}(1)$-subgroup of $\mathcal{L}$.

Proof. That it is an action follows from the cocycle condition for $d$. It is smooth because $d$ is a smooth bundle isomorphism over $L G_{\text {thin }}^{2}$. If $d$ is multiplicative, we compute for $p_{1} \in \mathcal{L}_{\tau_{1}}$ and $p_{2} \in \mathcal{L}_{\tau_{2}}$

$$
\left(p_{1} p_{2}\right) \cdot \varphi=d_{\tau_{1} \tau_{2},\left(\tau_{1} \tau_{2}\right) \circ \varphi}\left(p_{1} p_{2}\right)=d_{\tau_{1} \tau_{2},\left(\tau_{1} \circ \varphi\right)\left(\tau_{2} \circ \varphi\right)}\left(p_{1} p_{2}\right)=d_{\tau_{1}, \tau_{1} \circ \varphi}\left(p_{1}\right) d_{\tau_{2}, \tau_{2} \circ \varphi}\left(p_{2}\right) .
$$

The restriction of the $\operatorname{Diff}^{+}\left(S^{1}\right)$-action on $L G$ to constant loops is trivial. So if $p \in \mathcal{L}$ projects to a constant loop, we have $p \cdot \varphi=p$. In particular, $\operatorname{Diff}^{+}\left(S^{1}\right)$ acts trivially on $\mathrm{U}(1)$.

Definition 2.4. Suppose $\mathcal{L}$ is equipped with a fusion product $\lambda$ and a thin homotopy equivariant structure $d$. 
(a) We say that $d$ is compatible with $\lambda$, if for all paths $\left(\gamma_{1}, \gamma_{2}, \gamma_{3}\right) \in P\left(P G^{[3]}\right)$ such that the three paths $t \mapsto \gamma_{i}(t) \cup \gamma_{j}(t) \in L G$ are thin, the diagram

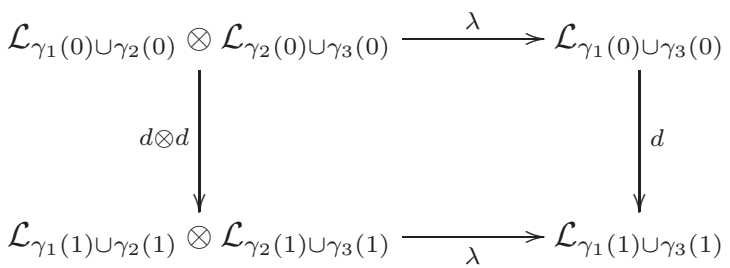

is commutative.

(b) We say that $d \underline{\text { symmetrizes }} \lambda$ if for all $\left(\gamma_{1}, \gamma_{2}, \gamma_{3}\right) \in P G^{[3]}$ and all $p \in \mathcal{L}_{\gamma_{1} \cup \gamma_{2}}$ and $p^{\prime} \in \mathcal{L}_{\gamma_{2} \cup \gamma_{3}}$

$$
d_{\gamma_{1} \cup \gamma_{3}, \overline{\gamma_{3}} \cup \overline{\gamma_{1}}}\left(\lambda\left(p \otimes p^{\prime}\right)\right)=\lambda\left(d_{\gamma_{2} \cup \gamma_{3}, \overline{\gamma_{3}} \cup \overline{\gamma_{2}}}\left(p^{\prime}\right) \otimes d_{\gamma_{1} \cup \gamma_{2}, \overline{\gamma_{2}} \cup \overline{\gamma_{1}}}(p)\right) .
$$

(c) We say that $d$ is fusive with respect to $\lambda$, if it is compatible and symmetrizing,

For (BD) we remark that if $r_{\pi} \in \mathcal{D i f f}^{+}\left(S^{1}\right)$ denotes the rotation by an angle of $\pi$, then $\left(\gamma_{i} \cup \gamma_{j}\right) \circ r_{\pi}=$ $\left(\overline{\gamma_{j}} \cup \overline{\gamma_{i}}\right)$; in particular, $\gamma_{i} \cup \gamma_{j}$ and $\overline{\gamma_{j}} \cup \overline{\gamma_{i}}$ are thin homotopic.

Example 2.5. Suppose $P$ is a principal U(1)-bundle over $G \times G$ with connection, such that there exists a connection-preserving isomorphism

$$
P_{g_{1}, g_{2}} \otimes P_{g_{1} g_{2}, g_{3}} \cong P_{g_{2}, g_{3}} \otimes P_{g_{1}, g_{2} g_{3}}
$$

over $G \times G \times G$. The subscript notation is that, for instance, $P_{g_{1} g_{2}, g_{3}}$ is the pullback of $P$ along the map $\left(g_{1}, g_{2}, g_{3}\right) \longmapsto\left(g_{1} g_{2}, g_{3}\right)$. The holonomy of $P$ is a smooth map $\eta: L G \times L G \longrightarrow \mathrm{U}(1)$ such that

$$
\eta\left(\tau_{1} \tau_{2}, \tau_{3}\right) \cdot \eta\left(\tau_{1}, \tau_{2}\right)=\eta\left(\tau_{1}, \tau_{2} \tau_{3}\right) \cdot \eta\left(\tau_{2}, \tau_{3}\right)
$$

for all $\tau_{1}, \tau_{2}, \tau_{3} \in L G$. Thus, $\eta$ is a 2 -cocycle in the smooth group cohomology of $L G$. It defines a group structure on $\mathcal{L}_{P}:=\mathrm{U}(1) \times L G$ via $\left(z_{1}, \tau_{1}\right) \cdot\left(z_{2}, \tau_{2}\right):=\left(z_{1} z_{2} \eta\left(\tau_{1}, \tau_{2}\right), \tau_{1} \tau_{2}\right)$, making $\mathcal{L}_{P}$ a central extension of $L G$. As the holonomy of a bundle, $\eta$ is a fusion map in the sense of Wal12b], i.e. it satisfies

$$
\eta\left(\gamma_{1} \cup \gamma_{3}, \gamma_{1}^{\prime} \cup \gamma_{3}^{\prime}\right)=\eta\left(\gamma_{1} \cup \gamma_{2}, \gamma_{1}^{\prime} \cup \gamma_{2}^{\prime}\right) \cdot \eta\left(\gamma_{2} \cup \gamma_{3}, \gamma_{2}^{\prime} \cup \gamma_{3}^{\prime}\right)
$$

for all $\left(\gamma_{1}, \gamma_{2}, \gamma_{3}\right),\left(\gamma_{1}^{\prime}, \gamma_{2}^{\prime}, \gamma_{3}^{\prime}\right) \in P G^{[3]}$. This is equivalent to the statement that the trivial fusion product $\lambda\left(\left(z_{12}, \gamma_{1} \cup \gamma_{2}\right) \otimes\left(z_{23}, \gamma_{2} \cup \gamma_{3}\right)\right):=\left(z_{12} z_{23}, \gamma_{1} \cup \gamma_{3}\right)$ is multiplicative. Likewise, we have the trivial thin homotopy equivariant structure $d\left(z, \tau_{0}\right):=\left(z, \tau_{1}\right)$ for each $\left(\tau_{0}, \tau_{1}\right) \in L G_{\text {thin }}^{2}$. It is fusive with respect to the trivial fusion product, and it is multiplicative with respect to the group structure defined by $\eta$ because the holonomy of a connection only depends on the thin homotopy class of a loop.

As a concrete example of this construction, one can take $G=\mathrm{U}(1)$ and $P$ the Poincaré bundle over $T:=\mathrm{U}(1) \times \mathrm{U}(1)$, equipped with its canonical connection. In differential cohomology, $P \in \hat{H}^{2}(T)$ is the cup product of the two projections $\mathrm{pr}_{1}, \mathrm{pr}_{2}: T \rightarrow \mathrm{U}(1)$ regarded as elements in $\hat{H}^{1}(T)$. This implies that the Poincaré bundle has an isomorphism (2.2). Its holonomy can be described in the following way. If $\tau \in L \mathrm{U}(1)$, let $n \in \mathbb{Z}$ be the winding number of $\tau$. One can find a smooth map $f: \mathbb{R} \rightarrow \mathbb{R}$ such that $f(t+1)=f(t)+n$ and $\tau=\mathrm{e}^{2 \pi \mathrm{i} f}$. For $\tau=\left(\tau_{1}, \tau_{2}\right) \in L T$, we get

$$
\eta\left(\tau_{1}, \tau_{2}\right)=\operatorname{Hol}_{P}(\tau)=\exp 2 \pi \mathrm{i}\left(n_{1} f_{2}(0)-\int_{0}^{1} f_{1}(s) f_{2}^{\prime}(s) \mathrm{d} s\right) .
$$

Using this formula, we obtain a central extension of $L \mathrm{U}(1)$, equipped with a multiplicative fusion product and a multiplicative and fusive thin homotopy equivariant structure. 
Example 2.6. Let $G$ be a compact, simple, connected, simply-connected Lie group, so that $L G$ has a universal central extension PS86. It can be realized by the following model of Mickelsson Mic87. We consider pairs $(\phi, z)$ where $\phi: D^{2} \rightarrow G$ is a smooth map that is radially constant near the boundary, and $z \in \mathrm{U}(1)$. We impose the following equivalence relation:

$$
(\phi, z) \sim\left(\phi^{\prime}, z^{\prime}\right) \Leftrightarrow \partial \phi=\partial \phi^{\prime} \quad \text { and } \quad z=z^{\prime} \cdot \mathrm{e}^{2 \pi \mathrm{i} S_{\mathrm{WZ}}(\Phi)} .
$$

Here, $\partial \phi \in L G$ denotes the restriction of $\phi$ to the boundary, and $\Phi: S^{2} \rightarrow G$ is the map defined on the northern hemisphere by $\phi$ (with the orientation-preserving identification) and on the southern hemisphere by $\phi^{\prime}$ (with the orientation-reversing identification). The symbol $S_{\mathrm{WZ}}$ stands for the WessZumino term defined as follows. Because $G$ is 2-connected, the map $\Phi$ can be extended to a smooth map $\tilde{\Phi}: D^{3} \rightarrow G$ defined on the solid ball. Then,

$$
S_{\mathrm{WZ}}(\Phi):=\int_{D^{3}} \tilde{\Phi}^{*} H \quad \text { with } \quad H:=\frac{1}{6}\langle\theta \wedge[\theta \wedge \theta]\rangle \in \Omega^{3}(G) .
$$

Here, $\theta \in \Omega^{1}(G, \mathfrak{g})$ is the left-invariant Maurer-Cartan from on $G$. The bilinear form $\langle-,-\rangle$ is normalized such that the closed 3 -form $H$ represents a generator $\mathrm{H}^{3}(G, \mathbb{Z}) \cong \mathbb{Z}$. Now, the total space of the principal $\mathrm{U}(1)$-bundle $\mathcal{L}_{G}$ is the set of equivalence classes of pairs $(\phi, z)$. The bundle projection sends $(\phi, z)$ to $\partial \phi \in L G$, and the $\mathrm{U}(1)$-action is given by multiplication in the $\mathrm{U}(1)$-component. The group structure on $\mathcal{L}_{G}$ turning it into a central extension is given by the Mickelsson product [Mic87]:

$$
\mathcal{L}_{G} \times \mathcal{L}_{G} \longrightarrow \mathcal{L}_{G}:\left(\left(\phi_{1}, z_{1}\right),\left(\phi_{2}, z_{2}\right)\right) \mapsto\left(\phi_{1} \phi_{2}, z_{1} z_{2} \cdot \exp 2 \pi \mathrm{i}\left(-\int_{D^{2}}\left(\phi_{1}, \phi_{2}\right)^{*} \rho\right)\right),
$$

where $\rho$ is defined by

$$
\rho:=\frac{1}{2}\left\langle\operatorname{pr}_{1}^{*} \theta \wedge \operatorname{pr}_{2}^{*} \bar{\theta}\right\rangle \in \Omega^{2}(G \times G) .
$$

The two differential forms $H$ and $\rho$ satisfy the identities

$$
\begin{aligned}
\Delta H & :=H_{g_{1}}-H_{g_{1} g_{2}}+H_{g_{2}}=\mathrm{d} \rho \\
\Delta \rho & :=\rho_{g_{1}, g_{2}}+\rho_{g_{1} g_{2}, g_{3}}-\rho_{g_{2}, g_{3}}-\rho_{g_{1}, g_{2} g_{3}}=0 .
\end{aligned}
$$

for all $g_{1}, g_{2}, g_{3} \in G$, where the subscripts are meant so that $\rho_{g_{1} g_{2}, g_{3}}$ is the pullback of $\rho$ along the map $\left(g_{1}, g_{2}, g_{3}\right) \longmapsto\left(g_{1} g_{2}, g_{3}\right)$. Eq. (2.5) assures that the Mickelsson product is well-defined on equivalence classes, and (2.6) implies its associativity.

A fusion product on $\mathcal{L}_{G}$ is defined as follows. For $\left(\gamma_{1}, \gamma_{2}, \gamma_{3}\right) \in P G^{[3]}$, we define

$$
\begin{aligned}
\lambda_{\gamma_{1}, \gamma_{2}, \gamma_{3}}:\left.\left.\mathcal{L}_{G}\right|_{\gamma_{1} \cup \gamma_{2}} \otimes \mathcal{L}_{G}\right|_{\gamma_{2} \cup \gamma_{3}} & \left.\longrightarrow \mathcal{L}_{G}\right|_{\gamma_{1} \cup \gamma_{3}} \\
\left(\phi_{12}, z_{12}\right) \otimes\left(\phi_{23}, z_{23}\right) & \mapsto\left(\phi_{13}, z_{12} z_{23} \cdot \mathrm{e}^{-2 \pi \mathrm{i} S_{\mathrm{WZ}}(\Psi)}\right),
\end{aligned}
$$

where $\phi_{13}: D^{2} \rightarrow G$ is an arbitrarily chosen smooth map with $\partial \phi_{13}=\gamma_{1} \cup \gamma_{3}$, and $\Psi: S^{2} \rightarrow G$ is obtained by trisecting $S^{2}$ along the longitudes $0, \frac{2 \pi}{3}$ and $\frac{4 \pi}{3}$, and prescribing $\Psi$ on each sector with the maps $\phi_{12}, \phi_{23}$ (with orientation-preserving identification) and $\phi_{13}$ (with orientation-reversing identification), respectively, see Figure 1 That (2.7) is independent of the choice of $\phi_{13}$ follows from the identity $S_{\mathrm{WZ}}(\Psi)=S_{\mathrm{WZ}}\left(\Psi^{\prime}\right) S_{\mathrm{WZ}}\left(\Phi_{13}\right)$ for Wess-Zumino terms, where $\Psi^{\prime}$ is obtained as described above but using a different map $\phi_{13}^{\prime}$ instead of $\phi_{13}$, and $\Phi_{13}$ is obtained in the way described earlier from $\phi_{13}$ and $\phi_{13}^{\prime}$. Definition (2.7) is also well-defined under the equivalence relation $\sim$ due to a similar identity for Wess-Zumino terms. Associativity follows from reparameterization invariance of the integral, and multiplicativity follows from the Polyakov-Wiegmann formula

$$
\mathrm{e}^{2 \pi \mathrm{i} S_{\mathrm{WZ}}\left(\Phi_{1}\right)} \cdot \mathrm{e}^{2 \pi \mathrm{i} S_{\mathrm{WZ}}\left(\Phi_{2}\right)}=\mathrm{e}^{2 \pi \mathrm{i} S_{\mathrm{WZ}}\left(\Phi_{1} \Phi_{2}\right)} \cdot \exp 2 \pi \mathrm{i}\left(\int_{\left(\Phi_{1}, \Phi_{2}\right)} \rho\right),
$$



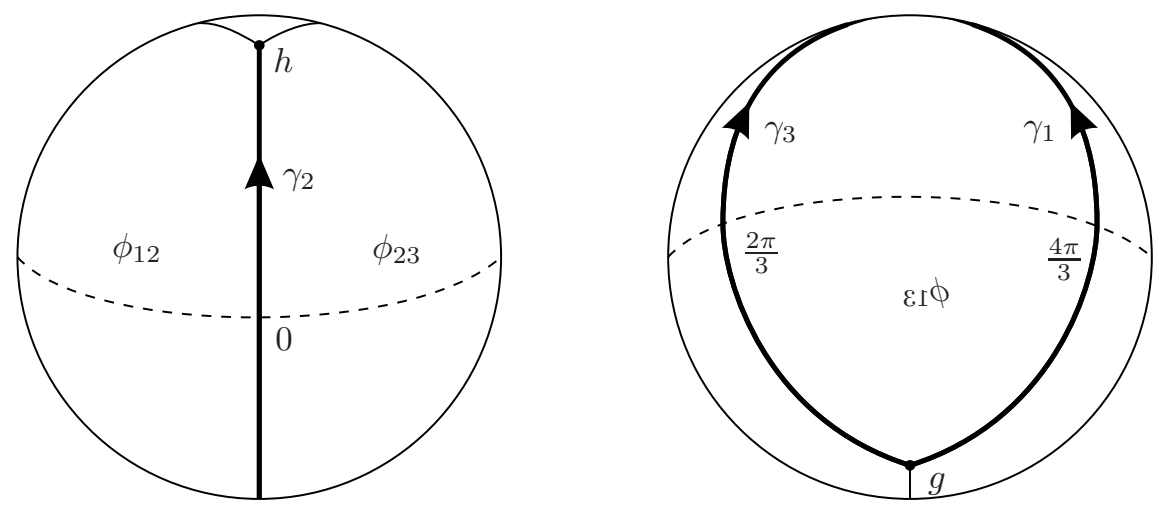

Figure 1: Front and back view to the map $\Psi$ in the definition of the fusion product over a triple $\left(\gamma_{1}, \gamma_{2}, \gamma_{3}\right) \in P G^{[3]}$ with common initial point $g$ and common end point $h$.

which in turn follows from (2.6).

A thin homotopy equivariant structure $d$ is defined as follows. Suppose $\left(\gamma_{0}, \gamma_{1}\right) \in L G_{\text {thin }}^{2}$. Then,

$$
d_{\gamma_{0}, \gamma_{1}}:\left.\left.\mathcal{L}_{G}\right|_{\gamma_{0}} \longrightarrow \mathcal{L}_{G}\right|_{\gamma_{1}}:\left(\phi_{0}, z_{0}\right) \longmapsto\left(\phi_{1}, z_{0} \cdot \mathrm{e}^{2 \pi \mathrm{i} S_{\mathrm{WZ}}\left(\Phi_{\gamma}\right)}\right),
$$

where $\phi_{1}: D^{2} \longrightarrow G$ is an arbitrarily chosen smooth map with $\partial \phi_{1}=\gamma_{1}$, and $\Phi_{\gamma}: S^{2} \longrightarrow G$ is the following map. On the polar caps $D^{2} \subseteq S^{2}$ we prescribe $\Phi$ with $\phi_{0}$ (around the north pole with orientation-reversing identification) and $\phi_{1}$ (around the south pole with orientation-preserving identification), and on the remaining cylinder $Z \cong[0,1] \times S^{1}$ by the homotopy $h_{\gamma}$ of a thin path $\gamma:[0,1] \rightarrow L G$ with $\gamma_{0}=\gamma(0)$ and $\gamma_{1}=\gamma(1)$. A different choice of $\phi_{1}$ gives an equivalent result. If another thin path $\gamma^{\prime}$ is chosen, then the two paths constitute a rank one loop in $L G$, i.e. a map $\Phi: S^{1} \times S^{1} \longrightarrow G$ of rank one, and we have to prove that $S_{\mathrm{WZ}}(\Phi)=0$. This follows as a special case of [Walb, Prop. 3.3.1]. In the next paragraphs we give an independent argument for the vanishing of Wess-Zumino terms for maps $\Phi: \Sigma \longrightarrow G$ of rank one, mapping a compact oriented surface $\Sigma$ to a connected, simple, simply-connected compact Lie group $G$.

Let $T$ be a maximal torus of $G, \mathfrak{t}$ its Lie algebra, and $\mathfrak{A} \subseteq \mathfrak{t}$ a closed Weyl alcove, a simplex with vertices $0=\mu_{0}, \ldots, \mu_{r}$, where $r$ is the rank of $G$. We let $F_{0} \subseteq \mathfrak{A}$ denote the closed face spanned by $\left\{\mu_{1}, \ldots, \mu_{r}\right\}, \mathfrak{A}_{0}:=\mathfrak{A} \backslash F_{0}$ its complement, and

$$
U:=\left\{h \exp \xi h^{-1} \mid h \in G \text { and } \xi \in \mathfrak{A}_{0}\right\}
$$

the corresponding open subset of $G$, which deformation retracts to $1 \in G$, see [Mei02, Lemma 5.1]. We show below that there exists $g \in G$ such that the left-translated map $\Phi_{g}: \Sigma \longrightarrow G, \Phi_{g}(x):=g \Phi(x)$, has its image contained in $U$. Due to the left-invariance of $H$, we have $S_{\mathrm{WZ}}(\Phi)=S_{\mathrm{WZ}}\left(\Phi_{g}\right)$. By composition with the deformation retract one obtains a rank-two map $\tilde{\Phi}_{g}: D \rightarrow U$ defined on a 3-dimensional manifold $D$ with $\partial D=\Sigma$, such that $\left.\tilde{\Phi}_{g}\right|_{\Sigma}=\Phi_{g}$. Thus,

$$
S_{\mathrm{WZ}}(\Phi)=S_{\mathrm{WZ}}\left(\Phi_{g}\right)=\int_{D} \tilde{\Phi}_{g}^{*} H=0 .
$$

Next we prove the existence of an appropriate left translating element $g$.

Recall that $g \in G$ is called regular if the conjugacy class of $g$ has maximal dimension. For the class of Lie groups under consideration, an element is regular if and only if it is conjugate to $\exp \xi$ 
for $\xi$ in the interior of $\mathfrak{A}$. In particular, the open subset $G^{\text {reg }}$ of regular elements is contained in $U$. We define $Q:=G \backslash U$, and obtain $Q \subseteq G \backslash G^{\mathrm{reg}}$. The set $G \backslash G^{\mathrm{reg}}$ is the disjoint union of finitely many submanifolds of $G$ of codimension $\geq 2$ [DK00, Page 137 Item $(\mathrm{g})]$. Let $Q_{\alpha}$ be one of these submanifolds. We consider the family $g \longmapsto \Phi_{g}$ of left translates, which, as a map $G \times \Sigma \longrightarrow G$, is a submersion and hence transverse to $Q_{\alpha}$. By the parametric transversality theorem Hir76, Chp. 3, Thm. 2.7] the set $G_{\alpha}:=\left\{g \in G \mid \Phi_{g}\right.$ is transverse to $\left.Q_{\alpha}\right\}$ is residual. But for dimensional reasons, a rank one map can only be transverse to a submanifold of codimension $\geq 2$ if its image does not intersect $Q_{\alpha}$, i.e. $G_{\alpha}=\left\{g \in G \mid \Phi_{g}(\Sigma) \cap Q_{\alpha}=\emptyset\right\}$. The intersection of finitely many residual sets is residual, in particular non-empty. Thus, there exists $g \in G$ such that $\Phi_{g}(\Sigma)$ does not intersect any of the submanifolds $Q_{\alpha}$, i.e. $\Phi_{g}(\Sigma) \subseteq U$. This finishes the proof that $S_{\mathrm{WZ}}(\Phi)=0$.

By now we have shown that the thin homotopy equivariant structure $d$ on $\mathcal{L}_{G}$ is welldefined as a bundle isomorphism over $L G_{\text {thin }}^{2}$. The cocycle condition follows from the identity $S_{\mathrm{WZ}}\left(\Phi_{\gamma_{2}}\right)+S_{\mathrm{WZ}}\left(\Phi_{\gamma_{1}}\right)=S_{\mathrm{WZ}}\left(\Phi_{\gamma_{2} \star \gamma_{1}}\right)$, if at $\gamma_{1}(1)=\gamma_{2}(0)$ the same extension $\phi: D^{2} \rightarrow G$ is chosen. Multiplicativity follows because in the Polyakov-Wiegmann formula (2.8) the error term vanishes, as the integral of the 2 -form $\rho$ along a thin path $(\gamma, \tau)$ through $L(G \times G)$ gives zero. Compatibility with the fusion product $\lambda$ can be seen by inspection of the occurring integrals. Finally, let us check in some more detail that $d$ symmetrizes $\lambda$. For $\left(\gamma_{1}, \gamma_{2}, \gamma_{3}\right) \in P G^{[3]}$, let $\phi_{12}, \phi_{23}, \phi_{13}: D^{2} \longrightarrow G$ such that $\lambda\left(\left(\phi_{12}, 1\right) \otimes\left(\phi_{23}, 1\right)\right)=\left(\phi_{13}, 1\right)$ holds. This means that $\mathrm{e}^{S_{\mathrm{WZ}}(\Psi)}=1$, with $\Psi: S^{2} \rightarrow G$ defined as shown in Figure 1. We have to check that

$$
\lambda\left(\left(\phi_{23}, 1\right) \cdot r_{\pi} \otimes\left(\phi_{12}, 1\right) \cdot r_{\pi}\right)=\left(\phi_{13}, 1\right) \cdot r_{\pi},
$$

where $r_{\pi} \in \mathcal{D i f f}^{+}\left(S^{1}\right)$ is the rotation by an angle of $\pi$. The definition of the thin homotopy equivariant structure implies $(\phi, z) \cdot r_{\pi}=\left(\phi \circ r_{\pi}, z\right)$, where on the right hand side $r_{\pi}$ is extended to a rotation of $D^{2}$. In order to check (2.9) we have to form the map $\Psi^{\prime}: S^{2} \rightarrow G$ using $\phi_{23} \circ r_{\pi}, \phi_{12} \circ r_{\pi}$ and $\phi_{13} \circ r_{\pi}$. By inspection, $\Psi^{\prime}=\Psi \circ r_{\pi}$, where now $r_{\pi}$ is extended to a rotation of $S^{2}$ about the front-back axis, compare Figures 1 and 2, Thus, $\mathrm{e}^{S_{\mathrm{WZ}}(\Psi)}=1$ and (2.9) holds.
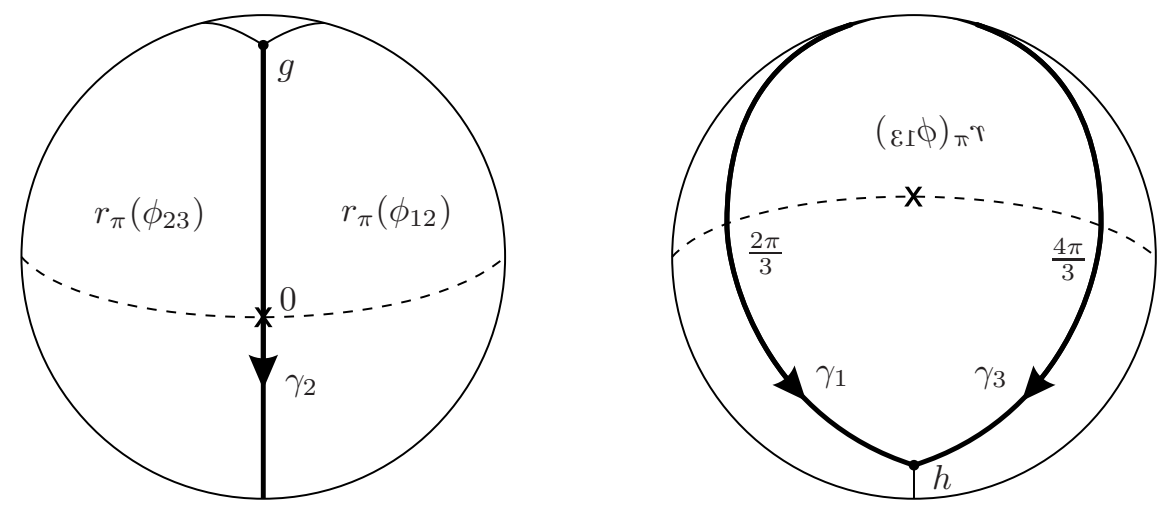

Figure 2: Front and back view to the map $\Psi^{\prime}$ defined as in Figure 1 but using the maps $\phi_{23} \circ r_{\pi}, \phi_{12} \circ r_{\pi}$ and $\phi_{13} \circ r_{\pi}$ instead of $\phi_{12}, \phi_{23}$ and $\phi_{13}$. The pictures are precisely the ones of Figure 1 rotated by an angle of $\pi$ around the axis that intersects $S^{2}$ at the two marked points. 


\section{Features of fusion and thin homotopy equivariance}

In this section we derive some consequences of the presence of a fusion product and a thin homotopy equivariant structure on a central extension. In particular, all results of this section hold for thin fusion extensions, and thus, by our main theorem, for transgressive central extensions.

\subsection{Flat loops and retraction}

Loops in the image of the map $b: P G \rightarrow L G: \gamma \mapsto \gamma \cup \gamma$ are called flat loops. Note that $b$ is a group homomorphism and that every constant loop is flat. Suppose $\phi$ is a smoothing map, i.e. it is a smooth map $\phi:[0,1] \longrightarrow[0,1]$ with $\phi(0)=0$ and $\phi(1)=1$, locally constant in a neighborhood of $\{0,1\}$, and smoothly homotopic to $\mathrm{id}_{[0,1]}$. Path retraction is the map

$$
[0,1] \times P G \longrightarrow P G:(t, \gamma) \mapsto \phi_{\gamma}(t)
$$

defined by $\phi_{\gamma}(t)(s):=\gamma(t \phi(s)) ; \phi$ is necessary to guarantee that $\phi_{\gamma}(t)$ has sitting instants. Clearly, $t \longmapsto b\left(\phi_{\gamma}(t)\right)$ is a thin path in $L G$; in particular, for every $t \in[0,1], b\left(\phi_{\gamma}(t)\right)$ is thin homotopic to $b(\gamma \circ \phi)$, which is in turn thin homotopic to $b(\gamma)$.

Proposition 3.1.1. Suppose $\mathcal{L}$ is a central extension of $L G$ equipped with a multiplicative fusion product $\lambda$ and a fusive thin homotopy equivariant structure $d$. Then, there exists a unique section $P G \longrightarrow \mathcal{L}: \gamma \longmapsto 1_{\gamma}$ along b such that $\lambda\left(1_{\gamma}, 1_{\gamma}\right)=1_{\gamma}$. It has the following properties:

(i) It is neutral with respect to fusion, i.e. $\lambda\left(p \otimes 1_{\gamma_{2}}\right)=p=\lambda\left(1_{\gamma_{1}} \otimes p\right)$ for all $p \in \mathcal{L}_{\gamma_{1} \cup \gamma_{2}}$.

(ii) It is a group homomorphism, i.e. $1_{\gamma_{1}} \cdot 1_{\gamma_{2}}=1_{\gamma_{1} \gamma_{2}}$.

(iii) It is retraction-invariant, i.e. $1_{\phi_{\gamma}(t)}=d_{b(\gamma), b\left(\phi_{\gamma}(t)\right)}\left(1_{\gamma}\right)$ for all $t \in[0,1]$.

Proof. Two sections $s, s^{\prime}: P G \rightarrow \mathcal{L}$ differ by a smooth map $\alpha: P G \rightarrow$ U(1), i.e., $s^{\prime}=\alpha s$. If the sections satisfy $\lambda(s, s)=s$ and $\lambda\left(s^{\prime}, s^{\prime}\right)=s^{\prime}$, we get $\alpha^{2}=\alpha$ and so $s=s^{\prime}$. For the existence, we notice that pulling back $\lambda$ along the diagonal map $P G \rightarrow P G^{[3]}$ shows that $b^{*} \mathcal{L}$ is trivializable. Let $s: P G \longrightarrow \mathcal{L}$ be any section. Then, there exists a unique smooth map $\alpha: P G \rightarrow \mathrm{U}(1)$ such that $\lambda(s \otimes s)=s \cdot \alpha$. Hence, $1_{\gamma}:=s(\gamma) \cdot \alpha(\gamma)$ has the desired property.

For (i) we have $\lambda\left(1_{\gamma_{1}} \otimes p\right)=\lambda\left(\lambda\left(1_{\gamma_{1}} \otimes 1_{\gamma_{1}}\right) \otimes p\right)=\lambda\left(1_{\gamma_{1}} \otimes \lambda\left(1_{\gamma_{1}} \otimes p\right)\right)$ using the associativity of the fusion product, and since $\lambda\left(1_{\gamma_{1}} \otimes-\right)$ is an isomorphism, we get $p=\lambda\left(1_{\gamma_{1}} \otimes p\right)$. Analogously we show neutrality from the right. For (ii) we have

$$
\lambda\left(\left(1_{\gamma_{1}} \cdot 1_{\gamma_{2}}\right) \otimes\left(1_{\gamma_{1}} \cdot 1_{\gamma_{2}}\right)\right)=\lambda\left(1_{\gamma_{1}} \otimes 1_{\gamma_{1}}\right) \cdot \lambda\left(1_{\gamma_{2}} \otimes 1_{\gamma_{2}}\right)=1_{\gamma_{1}} \cdot 1_{\gamma_{2}}
$$

using the multiplicativity of the fusion product; the uniqueness of the section then shows that $1_{\gamma_{1}}$. $1_{\gamma_{2}}=1_{\gamma_{1} \gamma_{2}}$. For (iii) we compute with the compatibility of Definition 2.4 (国)

$$
\lambda\left(d_{b(\gamma), b\left(\phi_{\gamma}(t)\right)}\left(1_{\gamma}\right) \otimes d_{b(\gamma), b\left(\phi_{\gamma}(t)\right)}\left(1_{\gamma}\right)\right)=d_{b(\gamma), b\left(\phi_{\gamma}(t)\right)}\left(\lambda\left(1_{\gamma} \otimes 1_{\gamma}\right)\right)=d_{b(\gamma), b\left(\phi_{\gamma}(t)\right)}\left(1_{\gamma}\right),
$$

from which the claim follows again from the uniqueness of the section.

In particular, the restriction of $\mathcal{L}$ to flat loops is canonically trivializable as a central extension of $P G$ by $\mathrm{U}(1)$. 


\subsection{Loop concatenation}

Let $\mathcal{L}$ be a central extension of $L G$ equipped with a fusion product. We start with the prototypical situation for loop concatenation: let $\gamma_{1}, \gamma_{2} \in P G$ be closed, i.e. loops with sitting instants. We have $\left(\gamma_{1}\right.$, id,$\left.\gamma_{2}\right) \in P G^{[3]}$. The concatenated loop is $\gamma_{1} \cup \gamma_{2}$. If $p_{1} \in \mathcal{L}_{\gamma_{1} \cup \text { id }}$ and $p_{2} \in \mathcal{L}_{\text {id } \cup \gamma_{2}}$, then

$$
\lambda\left(p_{1} \otimes p_{2}\right) \in \mathcal{L}_{\gamma_{1} \cup \gamma_{2}}
$$

this lifts loop concatenation from $L G$ to $\mathcal{L}$. In the following we use a fusive thin homotopy equivariant structure in order to generalize to arbitrary loops that admit concatenation (not necessarily with sitting instants).

We denote by $L G \times{ }_{G}^{\infty} L G$ the subset of $L G \times L G$ consisting of pairs $\left(\tau_{1}, \tau_{2}\right)$ such that $\tau_{1}(1)=\tau_{2}(1)$ and the concatenation $\overline{\tau_{2}} \star \tau_{1}$ is again a smooth loop. Thus, we have a well-defined map

$$
\text { con }: L G \times{ }_{G}^{\infty} L G \longrightarrow L G:\left(\tau_{1}, \tau_{2}\right) \longmapsto \overline{\tau_{2}} \star \tau_{1} .
$$

If we equip $L G \times{ }_{G}^{\infty} L G \subseteq L G \times L G$ with the subspace diffeology (i.e. a map $c: U \longrightarrow L G \times \times_{G}^{\infty} L G$ is a plot if and only if its extension to $L G \times L G$ is smooth), then con is smooth. Further, con is a group homomorphism.

We fix a smoothing map $\phi$ and construct new smooth maps $\phi_{1}, \phi_{2}:[0,1] \rightarrow[0,1]$ by setting:

$$
\phi_{1}(t):=\left\{\begin{array}{ll}
0 & 0 \leq t \leq \frac{1}{2} \\
\phi(2 t-1) & \frac{1}{2} \leq t \leq 1
\end{array} \quad \text { and } \quad \phi_{2}(t):= \begin{cases}\phi(2 t) & 0 \leq t \leq \frac{1}{2} \\
1 & \frac{1}{2} \leq t \leq 1 .\end{cases}\right.
$$

These cover well-defined smooth maps $\phi_{1}, \phi_{2}: S^{1} \rightarrow S^{1}$ that are smoothly homotopic to id $S_{S^{1}}$. Thus, if $\left(\tau_{1}, \tau_{2}\right) \in L G \times_{G}^{\infty} L G$ with $g:=\tau_{1}(1)=\tau_{2}(1)$, then $\tilde{\tau}_{k}:=\tau_{k} \circ \phi_{k}$ is thin homotopic to $\tau_{k}$ for $k=1,2$; furthermore $\tilde{\tau}_{1}=\left(\tau_{1} \circ \phi\right) \cup \operatorname{id}_{g}$ and $\tilde{\tau}_{2}=\operatorname{id}_{g} \cup\left(\tau_{2} \circ \phi\right)$.

Suppose we have elements $p_{k} \in \mathcal{L}_{\tau_{k}}$ for $k=1,2$. Using the thin homotopy equivariant structure, we define $\tilde{p}_{k}:=d_{\tau_{k}, \tilde{\tau}_{k}}\left(p_{k}\right)$, and form the fusion product

$$
\tilde{p}:=\lambda\left(\tilde{p}_{1} \otimes \tilde{p}_{2}\right) \in \mathcal{L}_{\left(\tau_{1} \circ \phi\right) \cup\left(\tau_{2} \circ \phi\right)} .
$$

The loop $\left(\tau_{1} \circ \phi\right) \cup\left(\tau_{2} \circ \phi\right)$ is thin homotopic to $\operatorname{con}\left(\tau_{1}, \tau_{2}\right)$. We obtain an element

$$
p:=d_{\left(\tau_{1} \circ \phi\right) \cup\left(\tau_{2} \circ \phi\right), \operatorname{con}\left(\tau_{1}, \tau_{2}\right)}(\tilde{p}) \in \mathcal{L}_{\operatorname{con}\left(\tau_{1}, \tau_{2}\right)} .
$$

Proposition 3.2.1. Suppose $\mathcal{L}$ is a central extension of $L G$ equipped with a fusion product $\lambda$ and a fusive thin homotopy equivariant structure $d$. Then, the assignment $\left(p_{1}, p_{2}\right) \mapsto p$ defined above is a smooth map

$$
\widetilde{\text { con }}: \operatorname{pr}_{1}^{*} \mathcal{L} \otimes \operatorname{pr}_{2}^{*} \mathcal{L} \rightarrow \operatorname{con}^{*} \mathcal{L}
$$

over $L G \times{ }_{G}^{\infty} L G$ that is independent of the choice of the smoothing function. If $\lambda$ and $d$ are multiplicative, then

$$
\widetilde{\operatorname{con}}\left(p_{1} \otimes p_{2}\right) \cdot \widetilde{\operatorname{con}}\left(p_{1}^{\prime} \otimes p_{2}^{\prime}\right)=\widetilde{\operatorname{con}}\left(p_{1} p_{1}^{\prime} \otimes p_{2} p_{2}^{\prime}\right)
$$

for all $p_{i} \in \mathcal{L}_{\tau_{i}}, p_{i}^{\prime} \in \mathcal{L}_{\tau_{i}^{\prime}}$ with $i=1,2$ and $\left(\tau_{1}, \tau_{2}\right),\left(\tau_{1}^{\prime}, \tau_{2}^{\prime}\right) \in L G \times_{G}^{\infty} L G$.

Proof. We show the independence of the smoothing function $\phi$. If $\phi^{\prime}$ is another smoothing function, then $\phi$ and $\phi^{\prime}$ are smoothly homotopic. We obtain loops $\tilde{\tau}_{1}^{\prime}$ and $\tilde{\tau}_{2}^{\prime}$ that are thin homotopic to $\tilde{\tau}_{1}$ and 
$\tilde{\tau}_{2}$, respectively. The diagram

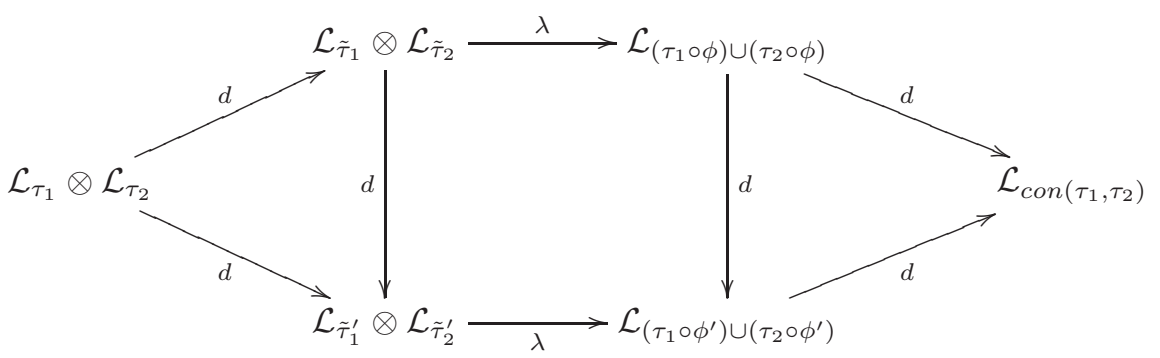

is commutative: the triangular diagrams commute due to the cocycle condition for $d$ (Definition 2.2 (国), and the rectangular diagram commutes due to the compatibility condition for $\lambda$ and $d$ (Definition 2.4 (国). This shows the independence of the choice of $\phi$.

Smoothness can be checked with fixed smoothing function and then follows from the smoothness of the thin structure and the fusion product. In order to see the multiplicativity, we notice that the thin paths from $\tau_{i}$ to $\tilde{\tau}_{i}$ give a thin path in $L(G \times G)$. Thus, the multiplicativity of $d$ and the one of the fusion product imply the claimed property.

\subsection{Disjoint-commutativity}

A loop $\tau \in L G$ is supported on an interval $I \subseteq S^{1}$ if $\tau(z)=1$ for all $z \in S^{1} \backslash I$.

Theorem 3.3.1. Suppose $\mathcal{L}$ is a central extension of $L G$ equipped with a multiplicative fusion product and a multiplicative, fusive thin homotopy equivariant structure. Then, $\mathcal{L}$ is disjoint commutative, i.e. if $\tau_{1}, \tau_{2} \in L G$ are supported on disjoint intervals, and $p_{1} \in \mathcal{L}_{\tau_{1}}$ and $p_{2} \in \mathcal{L}_{\tau_{2}}$, then $p_{1} \cdot p_{2}=p_{2} \cdot p_{1}$.

Theorem 3.3 .1 applies to the universal central extension $\mathcal{L}_{G}$ of a compact, simple, connected, simply-connected Lie group $G$, as seen in Example 2.6. In that case, disjoint commutativity was known to hold, verified by a direct calculation using the Mickelsson model [GF93, Lemma 3.1].

For the proof Theorem 3.3.1 we start with the following prototypical situation. Suppose $\tau_{1}, \tau_{2} \in$ $P G$ are loops based at $1 \in G$. Then, $\tau_{1} \cup \mathrm{id}_{1}$ and $\operatorname{id}_{1} \cup \tau_{2}$ are loops supported on disjoint intervals. In particular,

$$
\left(\tau_{1} \cup \operatorname{id}_{1}\right)\left(\operatorname{id}_{1} \cup \tau_{2}\right)=\left(\operatorname{id}_{1} \cup \tau_{2}\right)\left(\tau_{1} \cup \operatorname{id}_{1}\right) .
$$

Proposition 3.3.2. We have $p_{1} \cdot p_{2}=p_{2} \cdot p_{1}$ for all $p_{1} \in \mathcal{L}_{\tau_{1} \cup \mathrm{id}}$ and $p_{2} \in \mathcal{L}_{\mathrm{id}_{1} \cup \tau_{2}}$.

In order to prove this, we note that the multiplication in this particular situation coincides with the fusion product.

Lemma 3.3.3. $p_{1} \cdot p_{2}=\lambda\left(p_{1} \otimes p_{2}\right)$.

Proof. Proposition 3.1.1 (1ii) implies $1_{\mathrm{id}_{1}}=1$. Thus, over the triple $\left(\tau_{1}, \mathrm{id}_{1}, \mathrm{id}_{1}\right) \in P G^{[3]}$ we can write $p_{1}=\lambda\left(p_{1} \otimes 1\right)$. Likewise, over $\left(\mathrm{id}_{1}, \mathrm{id}_{1}, \tau_{2}\right)$ we have $p_{2}=\lambda\left(1 \otimes p_{2}\right)$. Multiplicativity of the fusion product then shows the claim:

$$
p_{1} \cdot p_{2}=\lambda\left(p_{1} \otimes 1\right) \cdot \lambda\left(1 \otimes p_{2}\right)=\lambda\left(p_{1} \cdot 1 \otimes 1 \cdot p_{2}\right)=\lambda\left(p_{1} \otimes p_{2}\right) .
$$


Proof of Proposition 3.3.2. That the thin homotopy equivariant structure symmetrizes the fusion product (Definition 2.4 (b) $)$ means, over the triple $\left(\tau_{1}, \mathrm{id}_{1}, \tau_{3}\right)$,

$$
d_{\tau_{1} \cup \tau_{3}, \overline{\tau_{3}} \cup \overline{\tau_{1}}}\left(\lambda\left(p_{1} \otimes p_{2}\right)\right)=\lambda\left(d_{\mathrm{id}_{1} \cup \tau_{3}, \overline{\tau_{3}} \cup \operatorname{id}_{1}}\left(p_{2}\right) \otimes d_{\tau_{1} \cup \mathrm{id}_{1}, \mathrm{id}_{1} \cup \overline{\tau_{1}}}\left(p_{1}\right)\right) .
$$

We can apply Lemma 3.3 .3 on the left (to the given data) and on the right (to the pair $\left(\overline{\tau_{3}}, \overline{\tau_{1}}\right.$ ) and the elements $d_{\mathrm{id}_{1} \cup \tau_{3}, \overline{\tau_{3}} \cup \mathrm{id}_{1}}\left(p_{2}\right)$ and $\left.d_{\tau_{1} \cup \mathrm{id}_{1}, \mathrm{id}_{1} \cup \overline{\tau_{1}}}\left(p_{1}\right)\right)$ and obtain

$$
d_{\tau_{1} \cup \tau_{3}, \overline{\tau_{3}} \cup \overline{\tau_{1}}}\left(p_{1} \cdot p_{2}\right)=d_{\mathrm{id}_{1} \cup \tau_{3}, \overline{\tau_{3}} \cup \operatorname{id}_{1}}\left(p_{2}\right) \cdot d_{\tau_{1} \cup \mathrm{id}_{1}, \mathrm{id}_{1} \cup \overline{\tau_{1}}}\left(p_{1}\right) .
$$

Now we want to use the multiplicativity of the thin homotopy equivariant structure. We claim that $\left(\left(\mathrm{id}_{1} \cup \tau_{3}, \tau_{1} \cup \mathrm{id}_{1}\right),\left(\overline{\tau_{3}} \cup \mathrm{id}_{1}, \operatorname{id}_{1} \cup \overline{\tau_{1}}\right)\right) \in L(G \times G)_{\text {thin }}^{2}$. Indeed, let $r_{-\pi}: S^{1} \rightarrow S^{1}$ denote the rotation by an angle of $-\pi$. Then, $\left(\operatorname{id}_{1} \cup \tau_{3}\right) \circ r_{-\pi}=\overline{\tau_{3}} \cup \mathrm{id}_{1}$ and $\left(\tau_{1} \cup \mathrm{id}_{1}\right) \circ r_{-\pi}=\mathrm{id}_{1} \cup \overline{\tau_{1}}$. Fixing a path $\varphi_{t}$ from $\varphi_{0}=\operatorname{id}_{S^{1}}$ to $\varphi_{1}=r_{-\pi}$ we obtain a path

$$
\left(\left(\operatorname{id}_{1} \cup \tau_{3}\right) \circ \varphi_{t},\left(\tau_{1} \cup \mathrm{id}_{1}\right) \circ \varphi_{t}\right)
$$

in $L(G \times G)$, which is thin as it factors through $\varphi_{t}(z) \in S^{1}$. Thus, the multiplicativity of the thin structure now implies $p_{1} \cdot p_{2}=p_{2} \cdot p_{1}$.

Proof of Theorem 3.3.1. We consider two general loops $\tau_{1}, \tau_{2} \in L G$ with disjoint supports $I, J \subseteq S^{1}$. There is an orientation-preserving diffeomorphism $\varphi: S^{1} \rightarrow S^{1}$ such that $\varphi^{-1}(I) \subseteq\left(\frac{1}{2}, 1\right)$ and $\varphi^{-1}(J) \subseteq\left(0, \frac{1}{2}\right)$. Then, $\tau_{1} \circ \varphi=\tau_{1}^{\prime} \cup \mathrm{id}_{1}$ and $\tau_{2} \circ \varphi=\mathrm{id}_{1} \cup \tau_{2}^{\prime}$, where $\tau_{1}^{\prime}\left(\mathrm{e}^{2 \pi \mathrm{i} t}\right)=\left(\tau_{1} \circ \varphi\right)\left(\mathrm{e}^{\pi \mathrm{i}(1-t)}\right)$ and $\tau_{2}^{\prime}\left(\mathrm{e}^{2 \pi \mathrm{i} t}\right)=\left(\tau_{2} \circ \varphi\right)\left(\mathrm{e}^{\pi \mathrm{i} t}\right)$. The map $\varphi$ is homotopic to $\mathrm{id}_{S^{1}}$ via a family $\varphi_{t}$ with $\varphi_{0}=\mathrm{id}_{S^{1}}$ and $\varphi_{1}=\varphi$. The path $\left(\tau_{1} \circ \varphi_{t}, \tau_{2} \circ \varphi_{t}\right)=\left(\tau_{1}, \tau_{2}\right) \circ \varphi_{t}$ in $L(G \times G)$ is thin. If $p_{1} \in \mathcal{L}_{\tau_{1}}$ and $p_{2} \in \mathcal{L}_{\tau_{2}}$, then $d_{\tau_{1}, \tau_{1}^{\prime} \cup \mathrm{id}_{1}}\left(p_{1}\right) \in \mathcal{L}_{\tau_{1}^{\prime} \cup \mathrm{id}_{1}}$ and $d_{\tau_{2}, \mathrm{id}_{1} \cup \tau_{2}^{\prime}}\left(p_{2}\right) \in \mathcal{L}_{\mathrm{id}_{1} \cup \tau_{2}^{\prime}}$. Proposition 3.3 .2 applies and

$$
d_{\tau_{1}, \tau_{1}^{\prime} \cup \mathrm{Uid}_{1}}\left(p_{1}\right) \cdot d_{\tau_{2}, \mathrm{id}_{1} \cup \tau_{2}^{\prime}}\left(p_{2}\right)=d_{\tau_{2}, \mathrm{id}_{1} \cup \tau_{2}^{\prime}}\left(p_{2}\right) \cdot d_{\tau_{1}, \tau_{1}^{\prime} \cup \mathrm{id}_{1}}\left(p_{1}\right) .
$$

We use the multiplicativity of the thin structure: on the left for the thin path $\left(\tau_{1}, \tau_{2}\right) \circ \varphi_{t}$ and on the right for the thin path $\left(\tau_{2}, \tau_{1}\right) \circ \varphi_{t}$, and obtain

$$
d_{\tau_{1} \tau_{2},\left(\tau_{1}^{\prime} \cup \operatorname{id}_{1}\right)\left(\mathrm{id}_{1} \cup \tau_{2}^{\prime}\right)}\left(p_{1} \cdot p_{2}\right)=d_{\tau_{2} \tau_{1},\left(\mathrm{id}_{1} \cup \tau_{2}^{\prime}\right) \cdot\left(\tau_{1}^{\prime} \cup \mathrm{id}_{1}\right)}\left(p_{2} \cdot p_{1}\right) .
$$

We have

$$
\left(\tau_{1}^{\prime} \cup \operatorname{id}_{1}\right)\left(\operatorname{id}_{1} \cup \tau_{2}^{\prime}\right)=\left(\operatorname{id}_{1} \cup \tau_{2}^{\prime}\right) \cdot\left(\tau_{1}^{\prime} \cup \operatorname{id}_{1}\right)=\tau_{1}^{\prime} \cup \tau_{2}^{\prime} \quad \text { and } \quad \tau_{1} \tau_{2}=\tau_{2} \tau_{1},
$$

and as $d_{\tau_{1} \tau_{2}, \tau_{1}^{\prime} \cup \tau_{2}^{\prime}}$ is an isomorphism, we get $p_{1} \cdot p_{2}=p_{2} \cdot p_{1}$.

\section{Integrable thin homotopy equivariant structures}

A thin homotopy equivariant structure on a central extension $\mathcal{L}$ can be induced from certain connections on (the underlying principal $\mathrm{U}(1)$-bundle of) $\mathcal{L}$.

\section{Definition 4.1.}

(a) A connection $\nu$ on $\mathcal{L}$ is called thin, if two thin paths $\gamma_{1}, \gamma_{2}$ with common initial point $\gamma_{1}(0)=\gamma_{2}(0)$ and common end point $\gamma_{1}(1)=\gamma_{2}(1)$ induce the same parallel transport, $p t_{\gamma_{1}}^{\nu}=p t_{\gamma_{2}}^{\nu}$.

(b) A thin connection $\nu$ on $\mathcal{L}$ is called superficial, if two loops $\tau_{1}, \tau_{2} \in L L G$ have the same holonomy whenever they are homotopic via a map $h:[0,1] \times S^{1} \times S^{1} \rightarrow G$ of rank at most two. 
Every thin connection $\nu$ induces a thin homotopy equivariant structure $d^{\nu}$ by $d_{\tau_{0}, \tau_{1}}^{\nu}:=p t_{\tau}^{\nu}$, where $\tau$ is an arbitrary thin path from $\tau_{0}$ to $\tau_{1}$, see Wal12c Lemma 3.1.5]. Superficiality is a property of connections in the image of transgression, and will be relevant in Section 5

Definition 4.2. Suppose $\mathcal{L}$ is equipped with a fusion product $\lambda$ and a thin connection $\nu$.

(a) $\nu$ is compatible with $\lambda$, if the bundle morphism $\lambda$ over $P G^{[3]}$ is connection-preserving.

(b) $\nu$ symmetrizes $\lambda$, if $d^{\nu}$ is symmetrizing.

(c) $\nu$ is fusive if it is compatible and symmetrizing.

If $\nu$ is a thin and fusive connection, then $d^{\nu}$ is fusive with respect to $\lambda$. The question whether or not a given thin homotopy equivariant structure can be induced from a thin connection gives rise to the following definition.

Definition 4.3. A thin homotopy equivariant structure $d$ is called thin structure, if there exists a superficial connection $\nu$ such that $d^{\nu}=d$. In the presence of a fusion product, it is called fusive thin structure if $\nu$ can be chosen fusive.

It remains to discuss multiplicativity in the setting of thin structures. This requires some attention, because it is not clear which multiplicativity condition one should impose on a connection $\nu$. First of all we note that every connection $\nu$ on $\mathcal{L}$ determines a 1 -form $\epsilon_{\nu} \in \Omega^{1}(L G \times L G)$ by

$$
\nu_{p_{1}}\left(X_{1}\right)+\nu_{p_{2}}\left(X_{2}\right)=\nu_{p_{1} p_{2}}\left(p_{1} X_{2}+X_{1} p_{1}\right)+\left.\epsilon_{\nu}\right|_{\tau_{1}, \tau_{2}}\left(\mathcal{X}_{1}, \mathcal{X}_{2}\right)
$$

for all $\tau_{1}, \tau_{2} \in L G, \mathcal{X}_{i} \in T_{\tau_{i}} L G$, as well as $p_{i} \in \mathcal{L}_{\tau_{i}}$ and $X_{i} \in T_{p_{i}} \mathcal{L}$ such that $p_{*}\left(X_{i}\right)=\mathcal{X}_{i}$. We call $\epsilon_{\nu}$ the error 1-form of $\nu$, it can be seen as a measure for the non-multiplicativity of $\nu$. We want to impose a multiplicativity condition for the connection $\nu$ by requiring that $\epsilon_{\nu}$ admits a path splitting in the following sense.

Definition 4.4. Let $X$ be a smooth manifold, $k \in \mathbb{N}$, and $\epsilon \in \Omega^{k}(L X)$. A path splitting of $\epsilon$ is a $k$-form $\kappa \in \Omega^{k}(P X)$ such that $\cup^{*} \epsilon=\operatorname{pr}_{2}^{*} \kappa-\operatorname{pr}_{1}^{*} \kappa$ on $P X^{[2]}$.

We formulate two additional conditions for path splittings that will be required, too. We recall that for every (Fréchet or diffeological) Lie group $K$ and every $k \in \mathbb{N}$ we have a complex

$$
0 \longrightarrow \Omega^{k}(K) \longrightarrow \Omega^{k}(K \times K) \longrightarrow \ldots
$$

whose differential $\Delta$ is the alternating sum over the pullbacks along the face maps of the simplicial manifold $B K$. A form in the kernel of $\Delta$ is called multiplicative.

For $K=L G$, (4.1) implies that $\epsilon_{\nu}$ is multiplicative: $\Delta \epsilon_{\nu}=0$.

For $K=P G$, it makes sense to require that path splittings of $\epsilon_{\nu}$ are multiplicative.

For the second condition, we recall from Section 3.1 that for $\gamma \in P X$ and a smoothing function $\phi$ we have a retraction $\phi_{\gamma}:[0,1] \rightarrow P X$ with $\phi_{\gamma}(0)=\operatorname{id}_{\gamma(0)}$ and $\phi_{\gamma}(1)=\gamma \circ \phi$. A 1 -form $\kappa \in \Omega^{1}(P X)$ is called contractible, if

$$
\int_{\phi_{\gamma}} \kappa=0
$$

for all $\gamma \in P X$ and some (and thus all) smoothing functions $\phi$. 
Before continuing, let us try to elucidate path splittings with the following example.

Example 4.5. For $S$ a $l$-dimensional compact oriented smooth manifold, possibly with boundary, we denote by ev : $C^{\infty}(S, X) \times S \rightarrow X$ the evaluation map, and let

$$
\tau_{S}: \Omega^{k}(X) \longrightarrow \Omega^{k-l}\left(C^{\infty}(S, X)\right): \rho \mapsto \int_{S} \operatorname{ev}^{*} \rho
$$

be the usual transgression of differential forms to the mapping space. For $\rho \in \Omega^{k+1}(X)$ we set $\epsilon:=\tau_{S^{1}}(\rho) \in \Omega^{k}(L X)$ and $\kappa:=\left.\tau_{[0,1]}(\rho)\right|_{P X} \in \Omega^{k}(P X)$. We claim:

- $\kappa$ is a path splitting for $\epsilon$. To see this, consider a path $\gamma:[0,1] \longrightarrow P G^{[2]}$, with $\gamma=\left(\gamma_{1}, \gamma_{2}\right)$ and the associated homotopies $h_{\gamma_{i}}:[0,1]^{2} \rightarrow G ; h_{\gamma_{i}}(t, s)=\gamma_{i}(t)(s)$. Note that

$$
\int_{\gamma_{i}} \kappa=\int_{0}^{1} \kappa_{\gamma_{i}(t)}\left(\partial_{t} \gamma_{i}(t)\right) \mathrm{d} t=\int_{0}^{1} \int_{0}^{1} \rho_{\gamma_{i}(t)(s)}\left(\partial_{s} \gamma_{i}(t)(s), \partial_{t} \gamma_{i}(t)(s)\right) \mathrm{d} s \mathrm{~d} t=-\int_{h_{\gamma_{i}}} \rho .
$$

We have

$$
\int_{\gamma} \cup^{*} \epsilon=\int_{0}^{1} \int_{0}^{1} \rho_{(\cup \circ \gamma)(t)(s)}\left(\partial_{s}(\cup \circ \gamma)(t)(z), \partial_{t}(\cup \circ \gamma)(t)(s)\right) \mathrm{d} s \mathrm{~d} t .
$$

Splitting the integral over $s$ in two parts (from 0 to $\frac{1}{2}$ ) and (from $\frac{1}{2}$ to 1 ), expressing it in terms of $\gamma_{1}$ and $\gamma_{2}$, and reparameterizing, we get

$$
\int_{\gamma} \cup^{*} \epsilon=\int_{h_{\gamma_{1}}} \rho-\int_{h_{\gamma_{2}}} \rho=\int_{\gamma} \operatorname{pr}_{2}^{*} \kappa-\operatorname{pr}_{1}^{*} \kappa .
$$

But two 1-forms coincide if their integrals along all paths coincide; this shows $\cup^{*} \epsilon=\operatorname{pr}_{2}^{*} \kappa-\operatorname{pr}_{1}^{*} \kappa$.

- If $X$ is a Lie group and $\rho$ is multiplicative, then $\kappa$ and $\epsilon$ are multiplicative, too. Indeed, $\tau_{S}$ is linear and natural with respect to smooth maps between smooth manifolds, and so commutes with the differential $\Delta$.

- If $k=1$, then $\kappa$ is contractible: if $\gamma \in P X$, then we have

$$
\int_{\phi_{\gamma}} \kappa=\int_{\phi_{\gamma}} \int_{[0,1]} \mathrm{ev}^{*} \rho=\int_{[0,1]^{2}}\left(\mathrm{ev} \circ\left(\phi_{\gamma} \times \mathrm{id}\right)\right)^{*} \rho=0
$$

because $\left(\mathrm{ev} \circ\left(\phi_{\gamma} \times \mathrm{id}\right)\right)(t, s)=\gamma(t \phi(s))$ is a rank one map.

One can show that the error 1-form $\epsilon_{\nu} \in \Omega^{1}(L G \times L G)$ of a fusive connection $\nu$ on $\mathcal{L}$ admits a path splitting, and for compact groups $G$ even a multiplicative path splitting. However, I do not know conditions that would guarantee the existence of a contractible path splitting. This constitutes our multiplicativity condition.

Definition 4.6. A multiplicative and fusive thin homotopy equivariant structure $d$ is called multiplicative and fusive thin structure, if there exists a fusive superficial connection $\nu$ with $d^{\nu}=d$, whose error 1-form $\epsilon_{\nu}$ admits a multiplicative and contractible path splitting.

Definition 4.7. Let $G$ be a Lie group and $L G=C^{\infty}\left(S^{1}, G\right)$ be its loop group.

(a) A thin fusion extension of $L G$ is a central extension

$$
1 \rightarrow \mathrm{U}(1) \longrightarrow \mathcal{L} \longrightarrow L G \longrightarrow 1
$$

together with a multiplicative fusion product and a multiplicative and fusive thin structure. 
(b) An isomorphism between thin fusion extensions is a smooth isomorphism between central extensions that is fusion-preserving and thin.

Thin fusion extensions form a category that we denote by $\mathcal{F} u \mathcal{E} \mathcal{E} x t^{t h}(L G)$.

Before coming to examples, we shall investigate the following interesting feature of a thin fusion extension (Proposition 4.9 below). We recall that the Lie algebra of $L G$ is $L \mathfrak{g}=C^{\infty}\left(S^{1}, \mathfrak{g}\right)$ with all operations defined pointwise. We denote the Lie algebra of a central extension $\mathcal{L}$ by $\mathfrak{l}$. It is a central extension

$$
0 \longrightarrow \mathbb{R} \longrightarrow \mathfrak{l} \stackrel{p_{*}}{\longrightarrow} L \mathfrak{g} \longrightarrow 0
$$

of Fréchet Lie algebras. We recall that a splitting is a continuous linear map $\delta: L \mathfrak{g} \longrightarrow \mathfrak{l}$ such that $p_{*} \circ \delta=\mathrm{id}_{L \mathfrak{g}}$. Every connection $\nu$ on $\mathcal{L}$ induces - via horizontal lift - a splitting $\delta_{\nu}$.

An element $\mathcal{X} \in L \mathfrak{g}$ is called linear loop, if there exist a smooth map $f: S^{1} \rightarrow \mathbb{R}$ and $X \in \mathfrak{g}$ such that $\mathcal{X}(z)=f(z) X$ for all $z \in S^{1}$. The linear loops span $L \mathfrak{g}$. Every linear loop $\mathcal{X}$ can be represented - as a tangent vector - by a thin curve, namely by $\gamma_{\mathcal{X}}: \mathbb{R} \longrightarrow L G: t \mapsto \exp (t \mathcal{X})$, with $\gamma_{\mathcal{X}}(t)(z)=\exp (t f(z) X)$. Note that $t \longmapsto\left(1, \gamma_{\mathcal{X}}(t)\right)$ is a smooth curve in $L G_{\text {thin }}^{2}$. Thus, a thin homotopy equivariant structure $d$ on $\mathcal{L}$ produces a smooth curve $d_{\mathcal{X}}: \mathbb{R} \rightarrow \mathcal{L}: t \mapsto d_{1, \gamma_{\mathcal{X}}(t)}(1)$.

Lemma 4.8. Suppose $\mathcal{L}$ is a central extension with a thin homotopy equivariant structure $d$. Let $\nu$ be a thin connection on $\mathcal{L}$ with $d^{\nu}=d$. Then, the splitting $\delta_{\nu}$ is $\operatorname{Diff}^{+}\left(S^{1}\right)$-equivariant and satisfies

$$
\delta_{\nu}(\mathcal{X})=\left.\frac{\mathrm{d}}{\mathrm{d} t}\right|_{0} d \mathcal{X}(t)
$$

for all linear loops $\mathcal{X}$.

Proof. We calculate

$$
\delta_{\nu}(\mathcal{X})=\left.\frac{\mathrm{d}}{\mathrm{d} t}\right|_{0} p t_{\gamma \mathcal{X}}^{\nu}(1, t)=\left.\frac{\mathrm{d}}{\mathrm{d} t}\right|_{0} d_{1, \gamma_{\mathcal{X}}(t)}(1)=\left.\frac{\mathrm{d}}{\mathrm{d} t}\right|_{0} d_{\mathcal{X}}(t)
$$

Next we consider $\varphi \in \operatorname{Diff}^{+}\left(S^{1}\right)$ and a linear loop $\mathcal{X}$. Then, $\exp (t \mathcal{X}) \circ \varphi=\exp (t(\mathcal{X} \circ \varphi))$ is thin homotopic to $\exp (t \mathcal{X})$, and we have

$$
d_{\mathcal{X}}(t) \cdot \varphi=d_{1, \exp (t \mathcal{X})}(1) \cdot \varphi=d_{\exp (t \mathcal{X}), \exp (t(\mathcal{X} \circ \varphi))}\left(d_{1, \exp (t \mathcal{X})}(1)\right)=d_{1, \exp (t(\mathcal{X} \circ \varphi))}(1)=d_{\mathcal{X} \circ \varphi}(t) .
$$

Taking derivatives and using (4.3), we obtain $\delta_{\nu}(\mathcal{X}) \cdot \varphi=\delta_{\nu}(\mathcal{X} \circ \varphi)$. Since the $\operatorname{Diff}^{+}\left(S^{1}\right)$-actions on $L \mathfrak{g}$ and $\mathfrak{l}$ are linear, and $L \mathfrak{g}$ is spanned by the linear loops, we conclude that $\delta_{\nu}$ is equivariant.

In case of a thin fusion extension, we obtain:

Proposition 4.9. Let $\mathcal{L}$ be a thin fusion extension with thin structure $d$. Then, there exists a unique splitting $\delta: L \mathfrak{g} \longrightarrow \mathfrak{l}$ of the Lie algebra extension, such that

$$
\delta(\mathcal{X})=\left.\frac{\mathrm{d}}{\mathrm{d} t}\right|_{0} d_{\mathcal{X}}(t)
$$

for all linear loops $\mathcal{X}$. Moreover, this splitting is $\operatorname{Diff}^{+}\left(S^{1}\right)$-equivariant.

Proof. Uniqueness follows because the linear loops span $L \mathfrak{g}$. Existence uses the existence of a thin connection $\nu$ on $\mathcal{L}$ such that $d=d^{\nu}$. The corresponding splitting $\delta_{\nu}$ has the claimed properties by Lemma 4.8 , 
In the remainder of this section we discuss three examples.

Example 4.10. The universal central extension $\mathcal{L}_{G}$ of a compact, simple, connected, simply-connected Lie group $G$ is a thin fusion extension. Since $\mathcal{L}_{G}$ is universal, it follows that every central extension of $L G$ is a thin fusion extension. In the model of Example 2.6 we obtain a connection $\nu$ by declaring its parallel transport along a path $\gamma:[0,1] \rightarrow L G$ via

$$
p t_{\gamma}:\left.\left.\mathcal{L}_{G}\right|_{\gamma(0)} \longrightarrow \mathcal{L}_{G}\right|_{\gamma(1)}:\left(\phi_{0}, z_{0}\right) \mapsto\left(\phi_{1}, z_{0} \cdot \mathrm{e}^{2 \pi \mathrm{i} S_{\mathrm{WZ}}\left(\Phi_{\gamma}\right)}\right),
$$

where $\phi_{1}: D^{2} \longrightarrow G$ is arbitrarily chosen such that $\partial \phi_{1}=\gamma(1)$, and $\Phi_{\gamma}$ is obtained from $\gamma, \phi_{0}$ and $\phi_{1}$ exactly as described in the definition of the thin homotopy equivariant structure $d$ on $\mathcal{L}_{G}$. In order to show that (4.4) defines a connection, it suffices to check (see [SW09, Theorem 5.4]):

(a) It is compatible with the concatenation of paths: this is obvious.

(b) It depends only on the thin homotopy class of the path, i.e. if $\gamma$ and $\gamma^{\prime}$ are paths in $L G$ with common initial loop and common end loop, and $h:[0,1]^{2} \rightarrow L G$ is a smooth map with $h(0, t)=$ $\gamma(t)$ and $h(1, t)=\gamma^{\prime}(t)$ (i.e. $h$ is a fixed-ends-homotopy) and with the property that $\int h^{*} \omega=0$ for all 2 -forms $\omega \in \Omega^{2}(L G)$ (i.e. $h$ is thin), then $p t_{\gamma_{1}}=p t_{\gamma_{2}}$. That this is the case can be seen by expressing the difference of the parallel transport maps (4.4) as the integral of $\omega:=\tau_{S^{1}}(H)$ along $h$, which thus vanishes.

(c) It depends smoothly on the path. This can be checked on smooth one-parameter family of paths, for which smoothness follows from the one of the integral of differential forms.

Thus, we have a connection $\nu$ on $\mathcal{L}_{G}$. It is straightforward to see that it is compatible with the fusion product $\lambda$. We have already seen in Example 2.6 that for a thin path $\gamma$ the parallel transport $p t_{\gamma}$ is independent of the choice of the thin path: this shows that $\nu$ is thin and induces $d$. It is also superficial: if two loops $\tau_{1}, \tau_{2} \in L L G$ are homotopic via a homotopy $h$, then the difference between their holonomies is given by $\exp 2 \pi \mathrm{i} \int_{h} H$, where $h$ is the homotopy. When $h$ has rank two, the difference vanishes. The curvature of $\nu$ is $\tau_{S^{1}}(H)$, and the error 1 -form is $\epsilon_{\nu}=\tau_{S^{1}}(\rho)$. Example 4.5 shows that $\epsilon_{\nu}$ has a multiplicative and contractible path splitting.

Example 4.11. For any Lie group $G$, the central extension $\mathcal{L}_{P}=\mathrm{U}(1) \times L G$ with the group structure defined from the holonomy $\eta$ of a principal $\mathrm{U}(1)$-bundle $P$ over $G \times G$, the trivial fusion product and the trivial thin structure (see Example 2.5), is a thin fusion extension. Indeed, an integrating connection $\nu$ can be obtained from any 2 -form $\omega \in \Omega^{2}(G)$ by $\nu:=\tau_{S^{1}}(\omega) \in \Omega^{1}(L G)$, e.g. $\omega=0$ works. It is easy to see that this gives a fusive superficial connection, and that it induces the trivial thin structure, see Wal12c, Proposition 3.1.8].

Example 4.12. We construct a central extension that cannot be equipped with the structure of a thin fusion extension. We work with $G=\mathrm{U}(1)$, and consider $\mathcal{L}=\mathrm{U}(1) \times L \mathrm{U}(1)$ equipped with the group structure induced by the following 2-cocycle $\eta: L \mathrm{U}(1) \times L \mathrm{U}(1) \rightarrow \mathrm{U}(1)$. If $\tau \in L \mathrm{U}(1)$, we denote by $n \in \mathbb{Z}$ the winding number of $\tau$, and find a smooth map $f: \mathbb{R} \rightarrow \mathbb{R}$ such that $f(t+1)=f(t)+n$ and $\tau=\mathrm{e}^{2 \pi \mathrm{i} f}$. Note that $f$ is determined by $\tau$ up to a shift by a constant $z \in \mathbb{Z}$. We define for $f: \mathbb{R} \rightarrow \mathbb{R}$ the average

$$
\widehat{f}:=\int_{0}^{1} f(s) \mathrm{d} s
$$

We define for $\alpha, \beta, \gamma \in \mathbb{R}$ :

$$
\eta\left(\tau_{1}, \tau_{2}\right)=\exp 2 \pi \mathrm{i}\left(\alpha \int_{0}^{1} f_{1}(s) f_{2}^{\prime}(s) \mathrm{d} s+\beta\left(n_{1} \widehat{f}_{2}+n_{2} \widehat{f}_{1}\right)+\gamma n_{1} f_{2}(0)\right) .
$$


It is straightforward to check that the cocycle condition is satisfied for arbitrary values of $\alpha, \beta, \gamma$. We remark that the 2 -cocycle $\eta$ is normalized, i.e. $\eta(1,1)=1$, for all parameters. We have to assure that $\eta$ is well-defined under shifting $f_{k}$ by integers $z_{k}$. We get

$$
\begin{aligned}
\int_{0}^{1}\left(f_{1}(s)+z_{1}\right)\left(f_{2}+z_{2}\right)^{\prime}(s) \mathrm{d} s & =\int_{0}^{1} f_{1}(s) f_{2}^{\prime}(s) \mathrm{d} s+z_{1} n_{2} \\
n_{1} \widehat{f_{2}+z_{2}}+n_{2} \widehat{f_{1}+z_{1}} & =n_{1} \widehat{f_{2}}+n_{2} \widehat{f_{1}}+n_{1} z_{2}+n_{2} z_{1} \\
n_{1}\left(f_{2}+z_{2}\right)(0) & =n_{1} f_{2}(0)+n_{1} z_{2} .
\end{aligned}
$$

Note that all differences that arise are integers. We see two options to obtained well-definedness:

1. We choose the constants $\alpha, \beta, \gamma$ such that all differences cancel: $\beta=-\gamma$ and $\alpha=\gamma$. Then we have an $\mathbb{R}$-family of well-defined 2 -cocycles. The corresponding central extensions are denoted by $\mathcal{L}_{\mathbb{R}}(\gamma)$.

2. We let $\alpha, \beta, \gamma \in \mathbb{Z}$ be arbitrary integers. Then, all differences vanish separately under exponentiation. This gives a $\mathbb{Z}^{3}$-family of well-defined 2-cocycles. The corresponding central extensions are denoted by $\mathcal{L}_{\mathbb{Z}}(\alpha, \beta, \gamma)$.

We have coincidence $\mathcal{L}_{\mathbb{R}}(k)=\mathcal{L}_{\mathbb{Z}}(k,-k, k)$ for all $k \in \mathbb{Z}$. We observe that $\mathcal{L}_{\mathbb{Z}}(-1,0,1)$ is the extension $\mathcal{L}_{P}$ of Examples 2.5 and 4.11 with $P$ the Poincaré bundle over $T=\mathrm{U}(1) \times \mathrm{U}(1)$. Further, $\mathcal{L}_{\mathbb{Z}}(1,1,-1)$ is the "basic central extension" of $L \mathrm{U}(1)$ [PS86, Prop. 4.7.5].

One can show that for all $\tau_{1}, \tau_{2}$, and all $\alpha, \beta, \gamma \in \mathbb{R}$ the following symmetry law holds:

$$
\left.\eta\left(\tau_{1}, \tau_{2}\right)=\exp 2 \pi \mathrm{i}\left(2 \alpha \int_{0}^{1} f_{1}(s) f_{2}^{\prime}(s) \mathrm{d} s-\alpha n_{2} n_{1}+(\gamma-\alpha) n_{1} f_{2}(0)-(\gamma+\alpha) n_{2} f_{1}(0)\right)\right) \eta\left(\tau_{2}, \tau_{1}\right) .
$$

This shows in the first place that the extensions $\mathcal{L}_{\mathbb{Z}}(0, \beta, 0)$ are commutative. Let $\phi:[0,1] \rightarrow[0,1]$ be a smoothing map. Define smooth maps $f_{1}, f_{2}:[0,1] \rightarrow \mathbb{R}$ by

$$
f_{1}(t):=\left\{\begin{array}{ll}
\phi(2 t) & 0 \leq t \leq \frac{1}{2} \\
0 & \frac{1}{2} \leq t \leq 1
\end{array} \quad \text { and } \quad f_{2}(t):= \begin{cases}1 & 0 \leq t \leq \frac{1}{2} \\
\phi(2 t-1) & \frac{1}{2} \leq t \leq 1\end{cases}\right.
$$

and extend them periodically with shifts by $n_{1}=n_{2}=-1$. The corresponding loops $\tau_{1}$ and $\tau_{2}$ have disjoint support. We have $f_{1} f_{2}^{\prime}=0, f_{1}(0)=f_{2}(0)=1$, and hence

$$
\eta\left(\tau_{1}, \tau_{2}\right)=\exp (2 \pi \mathrm{i} \alpha) \cdot \eta\left(\tau_{2}, \tau_{1}\right) .
$$

This shows that the central extensions $\mathcal{L}_{\mathbb{R}}(\gamma)$ with $\gamma \notin \mathbb{Z}$ do not satisfy the disjoint commutativity law of Theorem 3.3.1 and we conclude that they cannot be equipped with the structure of thin fusion extensions.

\section{Transgression-regression machine}

\subsection{Multiplicative bundle gerbes}

We use the theory of bundle gerbes and connections on those Mur96, Ste00, CJM02, Wal07b. We denote by $\mathcal{G} r b(X)$ and $\mathcal{G} r b^{\nabla}(X)$ the bicategories of bundle gerbes without and with connection over a smooth manifold $X$, respectively. Forgetting the connection is an essentially surjective, and in 
general neither full nor faithful functor $\mathcal{G} r b^{\nabla}(X) \rightarrow \mathcal{G} r b(X)$. The 1-morphisms are called (connectionpreserving) isomorphisms, and the 2-morphisms are called (connection-preserving) transformations. 2 -forms $\rho \in \Omega^{2}(X)$ can be considered as connections on the trivial bundle gerbe $\mathcal{I}$; as a bundle gerbe with connection it is denoted by $\mathcal{I}_{\rho}$.

Definition 5.1.1 ([तJM ${ }^{+}$05, Wal10] $)$. A multiplicative bundle gerbe with connection over a Lie group $G$ is a bundle gerbe $\mathcal{G}$ with connection over $G$, a multiplicative 2-form $\rho \in \Omega^{2}(G \times G)$, a connectionpreserving isomorphism

$$
\mathcal{M}: \mathcal{G}_{1} \otimes \mathcal{G}_{2} \longrightarrow \mathcal{G}_{12} \otimes \mathcal{I}_{\rho}
$$

over $G \times G$, and a connection-preserving transformation

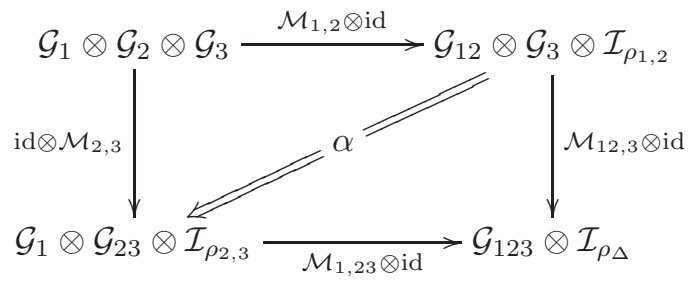

between isomorphisms over $G \times G \times G$, such that $\alpha$ satisfies a pentagon axiom over $G^{4}$.

Here our index convention is so that e.g. the index $(. .)_{i j, k}$ stands for the pullback along the map $\left(g_{i}, g_{j}, g_{k}\right) \longmapsto\left(g_{i} g_{j}, g_{k}\right)$. For instance, $\mathcal{G}_{i}=\operatorname{pr}_{i}^{*} \mathcal{G}$ and $\mathcal{G}_{12}$ is the pullback along the multiplication of $G$. Further, we have written $\rho_{\Delta}:=\rho_{1,2}+\rho_{12,3}=\rho_{2,3}+\rho_{1,23}$, with the equality coming from the multiplicativity of $\rho$, see (4.2). The pentagon axiom for $\alpha$ can be found in Wal10, Definition 1.3]. For later purpose, we need the following lemma.

Lemma 5.1.2. Suppose $G$ is connected. Let $\mathcal{G}$ be a bundle gerbe with connection over $G, \rho \in \Omega^{2}(G \times G)$ be a multiplicative 2-form, and $\mathcal{M}: \mathcal{G}_{1} \otimes \mathcal{G}_{2} \longrightarrow \mathcal{G}_{12} \otimes \mathcal{I}_{\rho}$ be a connection-preserving isomorphism, such that the set $X$ of connection-preserving transformations (5.1.1) is non-empty. Then, $X$ contains a unique element $\alpha$ that satisfies the pentagon axiom.

Proof. We pick some $\alpha \in X$. The pentagon axiom is an equality between two connection-preserving transformations over $G^{4}$. Set of connection-preserving transformations between two fixed connectionpreserving isomorphisms is a torsor over the group of locally constant $\mathrm{U}(1)$-valued maps. Thus, the pentagon axiom for $\alpha$ is satisfied up to a locally constant map $\epsilon: G^{4} \rightarrow \mathrm{U}(1)$. Since $G$ is connected, $\epsilon$ is constant; $\epsilon \in \mathrm{U}(1)$. We regard this constant as a locally constant map $\epsilon: G^{3} \rightarrow \mathrm{U}(1)$, and define a new element $\alpha^{\prime}:=\alpha \cdot \epsilon \in \mathcal{X}$. The pentagon axiom has five occurrences of $\alpha^{\prime}$ : three on one side and two on the other. Thus, four occurrences of $\epsilon$ cancel, and the remaining one compensates the error caused by $\alpha$; hence, $\alpha^{\prime}$ satisfies the pentagon axiom. Assume $\alpha, \alpha^{\prime} \in X$ satisfy the pentagon axiom. They differ by a locally constant map $\epsilon: G^{3} \longrightarrow \mathrm{U}(1)$, i.e. a constant. In the pentagon axioms for $\alpha$ and $\alpha^{\prime}$, this leads to $\epsilon^{3}=\epsilon^{2}$, i.e. $\epsilon=1$. Therefore, $\alpha=\alpha^{\prime}$.

If $(\mathcal{G}, \rho, \mathcal{M}, \alpha)$ and $\left(\mathcal{G}^{\prime}, \rho, \mathcal{M}^{\prime}, \alpha^{\prime}\right)$ are multiplicative bundle gerbes over $G$ with connections (with the same 2 -form $\rho$ ), a 1-morphism is a connection-preserving isomorphism $\mathcal{A}: \mathcal{G} \longrightarrow \mathcal{G}^{\prime}$ together with 
a connection-preserving transformation

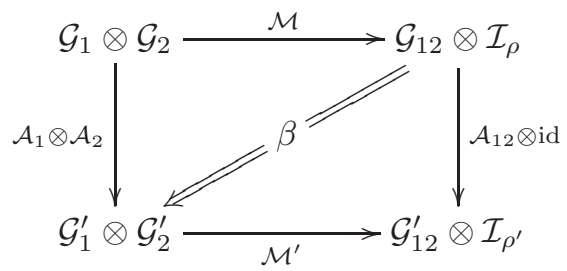

over $G \times G$ that satisfies a compatibility condition with respect to $\alpha$ and $\alpha^{\prime}$ over $G^{3}$, see [Wal10, Definition 1.7].

Lemma 5.1.3. Suppose $(\mathcal{G}, \rho, \mathcal{M}, \alpha)$ and $\left(\mathcal{G}^{\prime}, \rho, \mathcal{M}^{\prime}, \alpha^{\prime}\right)$ are multiplicative bundle gerbes with connection over a connected Lie group $G$, suppose $\mathcal{A}: \mathcal{G} \rightarrow \mathcal{G}^{\prime}$ is a connection-preserving isomorphism, and suppose $\beta$ is a connection-preserving transformation (5.1.2). Then, $\beta$ is compatible with $\alpha$ and $\alpha^{\prime}$.

Proof. We argue as in the proof of Lemma 5.1.2 The compatibility condition is an equality between two transformations over $G^{3}$, with four occurrences of $\beta$. Thus, it is satisfied up to a locally constant map $\epsilon: G^{3} \rightarrow \mathrm{U}(1)$, i.e. a constant. The two pentagon axioms for $\alpha$ and $\alpha^{\prime}$ over $G^{4}$ are related by $20=4 \cdot 5$ occurrences of $\beta$. As the pentagon axioms are satisfied, and the compatibility diagrams commute up to $\epsilon$, we obtain that five occurrences of $\epsilon$ have to cancel. This requires $\epsilon=1$.

If $(\mathcal{A}, \beta)$ and $\left(\mathcal{A}^{\prime}, \beta^{\prime}\right)$ are 1-morphisms between multiplicative bundle gerbes with connection, a 2-morphism is a connection-preserving transformation $\varphi: \mathcal{A} \Longrightarrow \mathcal{A}^{\prime}$ such that the diagram

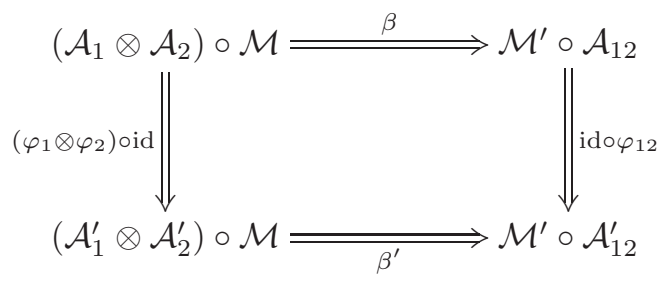

is commutative. With these definitions, multiplicative bundle gerbes with connection form a bicategory that we denote by $\mathcal{M u l t} \mathcal{G} r b^{\nabla}(G)$.

Multiplicative bundles gerbes without connections are defined analogously, without the 2 -form $\rho$ and without occurrences of trivial gerbes. We denote by $\mathcal{M u l t} \mathcal{G} r b(G)$ the bicategory of multiplicative bundle gerbes over $G$. We have the following classification result of $\left[\mathrm{CJM}^{+} 05\right]$ :

$$
\mathrm{h}_{0} \mathcal{M u l t} \mathcal{G} r b(G) \cong \mathrm{H}^{4}(B G, \mathbb{Z}),
$$

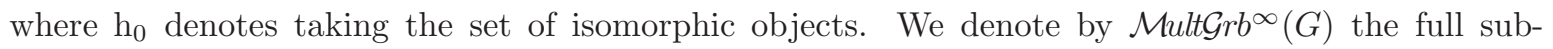
bicategory of $\mathcal{M u l t} \mathcal{G} r b(G)$ over those multiplicative bundle gerbes that admit connections. For compact Lie groups $G$, we have $\mathcal{M u l t} \mathcal{G} r b^{\infty}(G)=\mathcal{M u l t \mathcal { G } r b}(G)$ Wal10, Proposition 2.8]. All bicategories of multiplicative bundle gerbes are symmetric monoidal, under the tensor product of bundle gerbes, and (5.1.3) is an isomorphism between groups.

Example 5.1.4. The trivial gerbe $\mathcal{I}_{\omega}$ for any $\omega \in \Omega^{2}(G)$ carries multiplicative structures parameterized by principal U(1)-bundles $P$ with connection over $G \times G$ such that

$$
P_{1,2} \otimes P_{12,3} \cong P_{2,3} \otimes P_{1,23}
$$


via a coherent connection-preserving isomorphism, see [Wal10, Example 1.4]. In this case, the 2 -form is $\rho=\operatorname{curv}(P)-\omega$. For $G=\mathrm{U}(1)$, we have $\mathrm{H}^{4}(B \mathrm{U}(1), \mathbb{Z})=\mathrm{H}^{4}(K(\mathbb{Z}, 2), \mathbb{Z})=\mathbb{Z}$. In Wal10, Prop. $2.4]$ it is shown that there is an exact sequence

$$
0 \rightarrow \mathrm{H}^{3}(B \mathrm{U}(1), U(1)) \rightarrow \mathrm{h}_{0} \mathcal{M} \text { ult } \mathcal{G} r b^{\nabla}(\mathrm{U}(1)) \rightarrow \mathbb{Z}
$$

$\operatorname{As~}^{3}(B \mathrm{U}(1), U(1))=\mathrm{H}^{3}(K(\mathbb{Z}, 2), \mathrm{U}(1))=0$, we see that $\mathrm{h}_{0} \mathcal{M}$ ult $\mathcal{G} r b \nabla(\mathrm{U}(1)) \cong \mathrm{h}_{0} \mathcal{M}$ ult $\mathcal{G} r b(\mathrm{U}(1)) \cong \mathbb{Z}$. This $\mathbb{Z}$-family of multiplicative gerbes is obtained by taking $\omega=0$ and $P$ the Poincaré bundle over $T=\mathrm{U}(1) \times \mathrm{U}(1)$.

Example 5.1.5. Suppose $G$ is compact, simple and simply-connected. There exists a (up to connection-preserving isomorphisms) unique bundle gerbe $\mathcal{G}_{\text {bas }}$ with connection of curvature $H$, where $H \in \Omega^{3}(G)$ is the 3 -form of Example 2.6. it is called the basic gerbe. One can show that $\mathcal{G}_{\text {bas }}$ has a unique multiplicative structure [Wal10, Example 1.5], where $\rho \in \Omega^{2}(G \times G)$ is the 2-form of Example 2.6. There exist Lie-theoretical constructions of $\mathcal{G}_{\text {bas }}$ GR02, Mei02. Constructions of the corresponding multiplicative structures are notoriously difficult; one option is described in [Wal12a, Section 7]. For (non-simply connected) compact simple Lie groups, all multiplicative gerbes with connection are tabulated, and can be constructed via descent from their simply-connected covers GW09.

\subsection{Transgressive central extensions}

For every smooth manifold $X$, there is a transgression functor

$$
\mathscr{T}: \mathrm{h}_{1} \mathcal{G} r b^{\nabla}(X) \rightarrow \mathcal{B} u n(L X)
$$

with target the category of Fréchet principal U(1)-bundles over $L X$. The symbol $\mathrm{h}_{1}$ stands for passing from a bicategory to a category by identifying 2-isomorphic isomorphisms.

Transgression for gerbes has been defined by Gawędzki in terms of cocycles for Deligne cohomology Gaw88, and by Gawȩdzki-Reis for bundle gerbes GR02. Brylinski has defined transgression in terms of sheaves of categories Bry93. The functor (5.2.1) that we use here is an adaption of Brylinski's functor to bundle gerbes, and defined in Wal10. It is monoidal, and natural with respect to smooth maps $f: X \rightarrow X^{\prime}$ between smooth manifolds and the induced maps $L f: L X \rightarrow L X^{\prime}$ between their loop spaces. Furthermore, if $\rho \in \Omega^{2}(X), \mathcal{I}_{\rho}$ is the trivial bundle gerbe with connection $\rho$, then its transgression $\mathscr{T}_{\mathcal{I}_{\rho}}$ has a canonical trivialization $t_{\rho}: \mathscr{T}_{\mathcal{I}_{\rho}} \rightarrow \mathbf{I}$, where $\mathbf{I}$ is the trivial $\mathrm{U}(1)$-bundle over $L X$.

Suppose $(\mathcal{G}, \rho, \mathcal{M}, \alpha)$ is a multiplicative bundle gerbe with connection over $G$. Applying the transgression functor to $\mathcal{G}$, we obtain a Fréchet principal $\mathrm{U}(1)$-bundle $\mathcal{L}:=\mathscr{T}_{\mathcal{G}}$ over $L G$. Because transgression is functorial and monoidal, the transgression of the connection-preserving isomorphism $\mathcal{M}$ together with the trivialization $t_{\rho}$ give a bundle isomorphism

$$
\mathcal{L}_{1} \otimes \mathcal{L}_{2} \stackrel{\mathscr{T}_{\mathcal{M}}}{\longrightarrow} \mathcal{L}_{12} \otimes \mathscr{T}_{\mathcal{I}_{\rho}} \stackrel{\text { id } \otimes t_{\rho}}{\longrightarrow} \mathcal{L}_{12}
$$

over $L G \times L G$. It induces a binary operation on $\mathcal{L}$ that covers the group structure of $L G$. The existence of the transformation $\alpha$ implies under transgression the associativity of that binary operation. This equips $\mathcal{L}$ with the structure of a Fréchet Lie group Wal10, Theorem 3.1.7], making up a central extension

$$
1 \rightarrow \mathrm{U}(1) \rightarrow \mathcal{L} \rightarrow L G \rightarrow 1
$$


Definition 5.2.1. A central extension $\mathcal{L}$ of $L G$ is called transgressive, if there exists a multiplicative bundle gerbe with connection over $G$ whose transgression is isomorphic to $\mathcal{L}$ as a central extension.

In Walb a category $\mathcal{F} u s \mathcal{B} u n^{\nabla_{s f}}(L X)$ is considered with objects the Fréchet principal U(1)-bundles over $L X$ equipped with fusion products and fusive superficial connections, and morphisms the fusionpreserving, connection-preserving bundle morphisms. A construction in Walb, Section 4.2] lifts the transgression functor (5.2.1) to this category:

$$
\mathscr{T}^{\nabla}: \mathrm{h}_{1} \mathcal{G} r b^{\nabla}(X) \rightarrow \mathcal{F} u s \mathcal{B} u n^{\nabla f}(L X) .
$$

In case of a multiplicative bundle gerbe $\mathcal{G}$ with connection over $G$, this means in the first place that the underlying principal $\mathrm{U}(1)$-bundle of the central extension $\mathcal{L}$ is equipped with a fusion product $\lambda$ and with a fusive superficial connection $\nu$.

Under the lifted transgression functor, the transgression $\mathscr{T}_{\mathcal{I}_{\rho}}^{\nabla}$ of the trivial bundle gerbe with connection $\rho \in \Omega^{2}(X)$ is equipped with a fusion product and a connection, which under the trivialization $t_{\rho}: \mathscr{T}_{\mathcal{I}_{\rho}}^{\nabla} \rightarrow \mathbf{I}$ correspond to the trivial fusion product on $\mathbf{I}$ and the connection 1-form $\epsilon_{\nu}:=\tau_{S^{1}}(\rho) \in \Omega^{1}(L X)$ Wal11, Lemma 3.6]. Thus, the group structure of $\mathcal{L}$ is induced by the fusion-preserving, connection-preserving bundle morphism

$$
\mathcal{L}_{1} \otimes \mathcal{L}_{2} \stackrel{\mathscr{T}_{\mathcal{M}}^{\nabla}}{\longrightarrow} \mathcal{L}_{12} \otimes \mathscr{T}_{\mathcal{I}_{\rho}}^{\nabla} \stackrel{\mathrm{id} \otimes t_{\rho}}{\longrightarrow} \mathcal{L}_{12} \otimes \mathbf{I}_{\epsilon_{\nu}}
$$

This means (1) that $\epsilon_{\nu} \in \Omega^{1}\left(L G^{2}\right)$ is the error 1-form of $\nu$. By Example 4.5 $\kappa:=\left.\tau_{[0,1]}(\rho)\right|_{P(G \times G)}$ is a multiplicative and contractible path splitting for $\epsilon_{\nu}$. It means (2) that the fusion product is multiplicative, see Wala, Theorem 4.3.1].

Collecting all this data forces us to consider a category $\mathcal{F} u s \mathcal{E} x t^{\nabla}(L G)$ with:

- Objects: Central extensions of $L G$ equipped with a multiplicative fusion product, a fusive superficial connection, and a multiplicative, contractible path splitting of its error 1-form.

- Morphisms: Fusion-preserving, connection-preserving isomorphisms of central extensions, with the same error 1-form and the same path splitting on both sides.

The fact that transgression is a functor and monoidal implies that above procedure defines a monoidal functor

$$
\mathscr{M T}^{\nabla}: \mathrm{h}_{1} \mathcal{M} u l t \mathcal{G} r b^{\nabla}(G) \rightarrow \mathcal{F u s} \mathcal{E} x t^{\nabla}(L G)
$$

We can pass from superficial connections to thin structures in terms of an essentially surjective functor

$$
\text { th }: \mathcal{F} u s \mathcal{E} x t^{\nabla}(L G) \rightarrow \mathcal{F} u s \mathcal{E} x t^{t h}(L G)
$$

to the category of thin fusion extensions introduced in Section 4 Forgetting the fusion product and the thin structure gives another functor from $\mathcal{F} u s \mathcal{E} x t^{t h}(L G)$ to the category $\mathcal{E} x t(L G)$ of bare central extensions of $L G$. The composite

$$
\mathrm{h}_{1} \mathcal{M} \text { uultGrb } b^{\nabla}(G) \stackrel{\mathscr{M O}^{\nabla}}{\longrightarrow} \mathcal{F} u s \mathcal{E} x t^{\nabla}(L G) \stackrel{t h}{\longrightarrow} \mathcal{F} u s \mathcal{E} x t^{t h}(L G) \longrightarrow \mathcal{E} x t(L G)
$$


is the procedure from the beginning of the present subsection: the transgression of a multiplicative bundle gerbe with connection to a central extension. Thus, we obtain the following result, constituting the first part of Theorem $\mathrm{A}$.

Proposition 5.2.2. A central extension is transgressive only if it can be equipped with the structure of a thin fusion extension.

The functor (5.2.3) has a version when the connections on both sides are dropped, at the price that it only exists as map, not as a functor.

Proposition 5.2.3. There exists a unique map $\mathscr{M T}: \mathrm{h}_{0} \mathcal{M} u l t \mathcal{G} r b^{\infty}(G) \rightarrow \mathrm{h}_{0} \mathcal{F}$ usExt ${ }^{\text {th }}(L G)$ such that the diagram

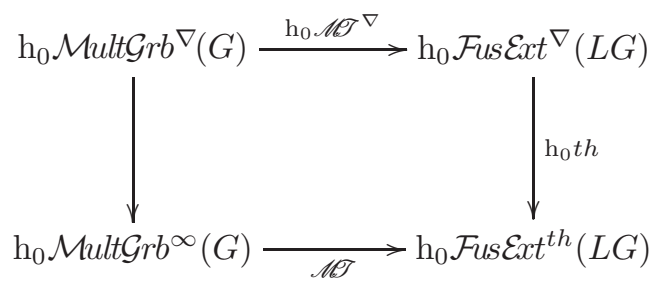

is commutative.

Proof. Uniqueness is clear as the vertical map on the left is surjective (by definition of $\mathcal{M u l t} \mathcal{G} r b^{\infty}(G)$ ). For the existence, we prove that the thin fusion extensions one gets from different choices of connections on the same multiplicative bundle gerbe are isomorphic.

Let $\mathcal{G}$ be a multiplicative bundle gerbe over $G$ with two connections, say $\lambda_{1}$ and $\lambda_{2}$, with corresponding thin fusion extensions $\mathcal{L}_{1}$ and $\mathcal{L}_{2}$, respectively. In [NW13, Proposition 5.1.3] we have have constructed an isomorphism $\varphi: \mathcal{L}_{1} \longrightarrow \mathcal{L}_{2}$ between central extensions. It was defined as the composition $\varphi:=\left(\right.$ id $\left.\otimes t_{\beta}\right) \circ \mathscr{T}_{\text {id }}^{\nabla}$, where $\beta \in \Omega^{2}(G)$ and $\operatorname{id}_{\epsilon}: \mathcal{G}_{\lambda_{1}} \rightarrow \mathcal{G}_{\lambda_{2}} \otimes \mathcal{I}_{\beta}$ is a connection-preserving isomorphism. The transgressed isomorphism $\mathscr{T}_{\mathrm{id}_{\epsilon}}^{\nabla}$ and the canonical trivialization $t_{\beta}: \mathscr{T}_{\mathcal{I}_{\beta}}^{\nabla} \longrightarrow \mathbf{I}_{\tau_{S^{1}}(\beta)}$ are fusion-preserving; this shows that $\varphi$ is fusion-preserving. As a connection on the trivial bundle, the 1-form $\tau_{S^{1}}(\beta)$ induces the trivial thin structure [Wal12c, Proposition 3.2.3]; so that $t h\left(t_{\beta}\right)$ is a thin bundle morphism. Hence, $\varphi$ is an isomorphism of thin fusion extensions.

Example 5.2.4. We consider the transgression of the trivial gerbe $\mathcal{I}_{\omega}$ equipped with a multiplicative structure defined by a principal U(1)-bundle $P$ with connection over $G \times G$ (see Example 5.1.4). The trivialization $t_{\omega}: \mathscr{T}_{\mathcal{I}_{\omega}}^{\nabla} \longrightarrow \mathrm{U}(1) \times L G$ induces an isomorphism between the transgressive thin fusion extension $\mathscr{T}_{\mathcal{I}_{\omega}}^{\nabla}$ and the thin fusion extension $\mathcal{L}_{P}$ of Examples 2.5 and 4.11. In particular, $\mathcal{L}_{P}$ is transgressive.

Example 5.2.5. Let $G$ be a compact, simple, simply-connected Lie group, let $\mathcal{G}_{\text {bas }}$ be the basic gerbe over $G$ (Example 5.1.5), and let $\mathcal{L}_{G}$ be the universal central extension of $L G$ (Examples 2.6 and 4.10). There is an isomorphism

$$
\varphi: \mathcal{L}_{G} \longrightarrow \mathscr{T}_{\mathcal{G}_{\text {bas }}}^{\nabla}
$$

of central extensions that is fusion-preserving and thin, and so establishes an isomorphism between thin fusion extensions. In particular, the universal central extension $\mathcal{L}_{G}$ is transgressive. The isomorphism $\varphi$ is defined by

$$
\varphi(\phi, z)=\partial \mathcal{T} \cdot z \cdot \exp 2 \pi \mathrm{i}\left(-\int_{D^{2}} \omega\right) .
$$


Here, $\mathcal{T}: \phi^{*} \mathcal{G}_{\text {bas }} \longrightarrow \mathcal{I}_{\omega}$ is an arbitrarily chosen trivialization of $\phi^{*} \mathcal{G}_{\text {bas }}$ over $D^{2}$ and $\partial \mathcal{T}$ denotes its restriction to the boundary; the latter is a trivialization of $\partial \phi^{*} \mathcal{G}_{\text {bas }}$ over $S^{1}$, constituting an element in $\mathscr{T}_{\mathcal{G}_{\text {bas }}}^{\nabla}$ over the loop $\partial \phi$. It is straightforward to see that $\varphi$ is well-defined, U(1)-equivariant, fusionpreserving, and a group homomorphism; see Wala, Section 4.3] for details. In order to see that $\varphi$ is thin, we consider the thin connection $\nu$ on $\mathcal{L}_{G}$ that integrates the thin homotopy equivariant structure $d$ (see Example 4.10), and show the stronger statement that $\varphi$ is connection-preserving. Indeed, if $\gamma:[0,1] \longrightarrow L G$ is a path, and $\phi_{0}, \phi_{1}: D^{2} \longrightarrow G$ are smooth maps with $\partial \phi_{0}=\gamma(0)$ and $\partial \phi_{1}=\gamma(1)$, then $p t_{\gamma}^{\nu}\left(\phi_{0}, 1\right)=\left(\phi_{1}, \mathrm{e}^{2 \pi \mathrm{i} S_{\mathrm{WZ}}\left(\Phi_{\gamma}\right)}\right)$. Let $\mathcal{T}_{0}, \mathcal{T}_{1}$ be trivializations of $\phi_{0}^{*} \mathcal{G}_{\text {bas }}$ and $\phi_{1}^{*} \mathcal{G}_{\text {bas }}$, respectively, and let $\tilde{\nu}$ denote the connection on $\mathscr{T}_{\mathcal{G}_{\text {bas }}}^{\nabla}$. Employing the definition of the transgression functor, see Walb, Section 4.3], the parallel transport in $\mathscr{T}_{\mathcal{G}_{b a s}}^{\nabla}$ is $p t_{\gamma}^{\tilde{\nu}}\left(\partial \mathcal{T}_{0}\right)=\partial \mathcal{T}_{1} \cdot \mathcal{A}_{\mathcal{G}_{\text {bas }}}\left(h_{\gamma}, \mathcal{T}_{0}, \mathcal{T}_{1}\right)$, where the latter term is the surface holonomy of $\mathcal{G}_{\text {bas }}$ with the trivializations as boundary conditions. In the present case of a 2-connected Lie group, it can be computed via the 3 -form $H$ and the two 2 -forms $\omega_{0}, \omega_{1}$ of the trivializations $\mathcal{T}_{0}, \mathcal{T}_{1}$, namely as

$$
\mathcal{A}_{\mathcal{G}_{\text {bas }}}\left(h_{\gamma}, \mathcal{T}_{0}, \mathcal{T}_{1}\right)=\exp 2 \pi \mathrm{i}\left(S_{\mathrm{WZ}}\left(\Phi_{\gamma}\right)+\int_{D^{2}} \omega_{0}-\int_{D^{2}} \omega_{1}\right)
$$

see [Wal07a, Proposition 3.1.4 (iii)]. Now we compute

$$
\begin{aligned}
p t_{\gamma}\left(\varphi\left(\phi_{0}, 1\right)\right)=p t_{\gamma}\left(\partial \mathcal{T}_{0}\right) \cdot \exp 2 \pi \mathrm{i}\left(-\int_{D^{2}} \omega_{0}\right)=\partial \mathcal{T}_{1} \cdot \exp 2 \pi \mathrm{i} & \left(S_{\mathrm{WZ}}\left(\Phi_{\gamma}\right)-\int_{D^{2}} \omega_{1}\right) \\
& =\varphi\left(\phi_{1}, \mathrm{e}^{2 \pi \mathrm{i} S_{\mathrm{WZ}}\left(\Phi_{\gamma}\right)}\right)=\varphi\left(p t_{\gamma}^{\nu}\left(\phi_{0}, 1\right)\right) .
\end{aligned}
$$

This shows that $\varphi$ commutes with the parallel transport along arbitrary paths; hence, $\varphi$ is connectionpreserving, in particular thin.

\subsection{Regression and equivalence result}

By the main result of [Walb], the lifted transgression functor (5.2.2) is an equivalence of categories, and has for fixed $x \in X$ a canonical inverse functor

$$
\mathscr{R}_{x}^{\nabla}: \mathcal{F} u s \mathcal{B} u n^{\nabla_{s f}}(L X) \rightarrow \mathrm{h}_{1} \mathcal{G} r b^{\nabla}(X)
$$

called regression. We need the following result about the regression of trivial bundles, which is explained in Appendix A. If $\epsilon \in \Omega^{1}(L X)$ is a superficial connection on the trivial bundle over $L X$, and fusive with respect to the trivial fusion product, then every path splitting $\kappa \in \Omega^{1}(P X)$ defines a connection-preserving isomorphism $\mathcal{T}_{\kappa}: \mathscr{R}^{\nabla}\left(\mathbf{I}_{\epsilon}\right) \rightarrow \mathcal{I}_{\rho_{\kappa}}$ between the regression of $\mathbf{I}_{\epsilon}$ and the trivial bundle gerbe $\mathcal{I}$ over $X$ equipped with a connection 2-form $\rho_{\kappa} \in \Omega^{2}(X)$ defined from $\kappa$.

If $X$ is a group, we always choose $x=1$ and omit the index. We use the functor $\mathscr{R}^{\nabla}=\mathscr{R}_{1}^{\nabla}$ in order to construct a regression functor

$$
\mathscr{M R}^{\nabla}: \mathcal{F u s} \operatorname{Ext}^{\nabla}(L G) \rightarrow \mathrm{h}_{1} \mathcal{M u l t G}^{\nabla} b^{\nabla}(G)
$$

defined on the category $\mathcal{F} u s \mathcal{E} x t^{\nabla}(L G)$ introduced in the previous section. Suppose $\mathcal{L}$ is a central extension of $L G$ equipped with a multiplicative fusion product $\lambda$, a fusive superficial connection, and a multiplicative path splitting $\kappa$ of its error 1 -form $\epsilon$ (for the definition of $\mathscr{M} \mathscr{R} \nabla$ we do not need that $\kappa$ is contractible). The group structure defines over $L G \times L G$ a connection-preserving, fusion-preserving bundle isomorphism

$$
\mu: \mathcal{L}_{1} \otimes \mathcal{L}_{2} \longrightarrow \mathcal{L}_{12} \otimes \mathbf{I}_{\epsilon},
$$


and the associativity of the group structure implies a commutative diagram over $L G^{3}$.

We let $\mathcal{G}:=\mathscr{R}^{\nabla}(\mathcal{L})$ be the regressed bundle gerbe over $G$ with connection. Over $G \times G$ we consider the connection-preserving isomorphism $\mathcal{M}$ defined as

$$
\mathcal{G}_{1} \otimes \mathcal{G}_{2} \stackrel{\mathscr{R}^{\nabla}(\mu)}{\longrightarrow} \mathcal{G}_{12} \otimes \mathscr{R}^{\nabla}\left(\mathbf{I}_{\epsilon}\right) \stackrel{\mathrm{id} \otimes \mathcal{T}_{\kappa}}{\longrightarrow} \mathcal{G}_{12} \otimes \mathcal{I}_{\rho_{\kappa}} .
$$

The various pullbacks of $\mathcal{M}$ to $G \times G \times G$ constitute the outer arrows of the following diagram in the category $\mathrm{h}_{1} \mathcal{G} r b \nabla(G \times G \times G)$ :

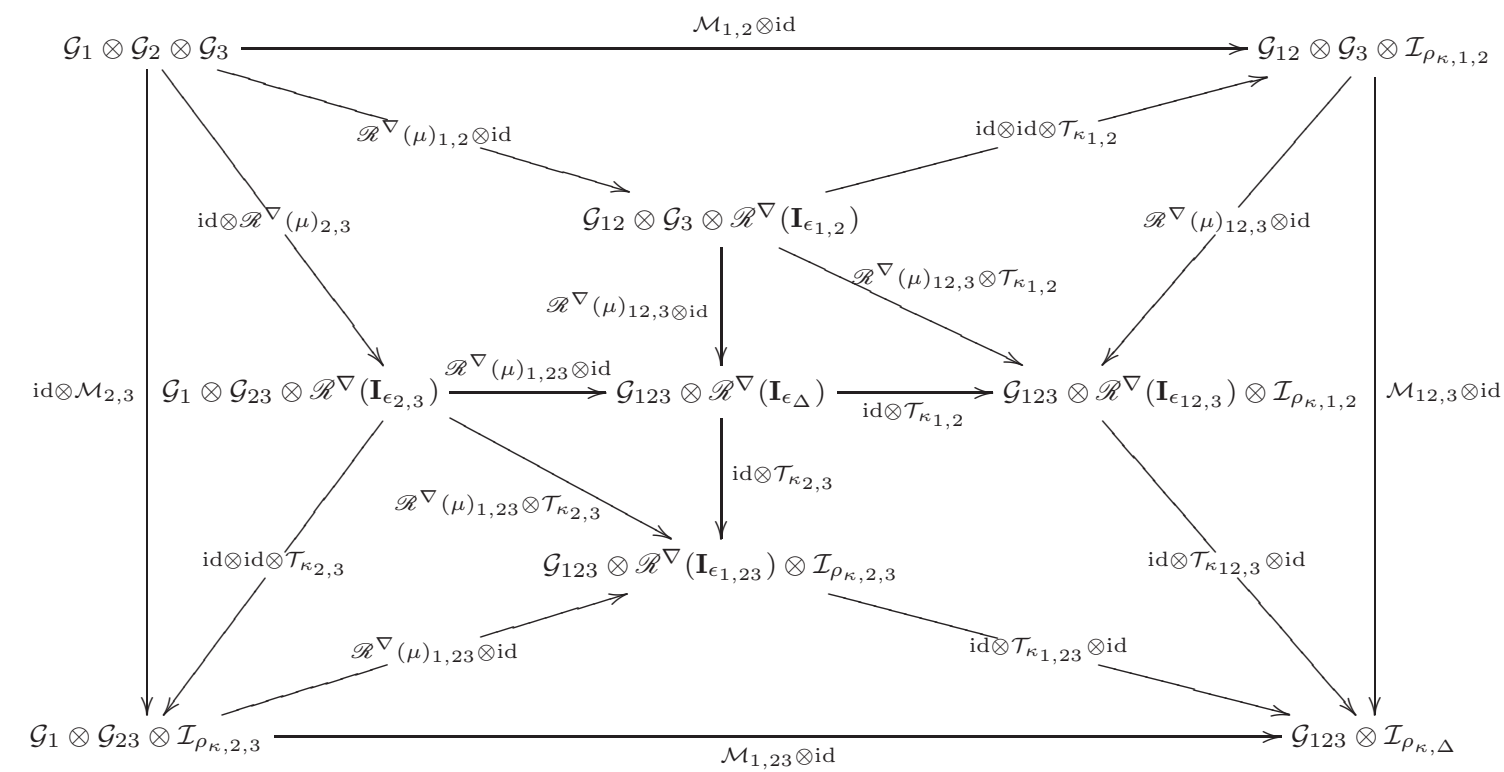

All triangular subdiagrams commute obviously. There remain two four-sided subdiagrams whose commutativity is to check. The one that touches the upper left corner commutes due to the commutative diagram for $\mu$ over $L G^{3}$ and the fact that $\mathscr{R}^{\nabla}$ is a functor. The one that touches the lower right corner commutes due to the multiplicativity of $\kappa$ and the additivity of the trivialization $\mathcal{T}_{\kappa}$ shown in Lemma A.2

The commutativity of the diagram in $\mathrm{h}_{1} \mathcal{G} r b^{\nabla}\left(G^{3}\right)$ implies the existence of a connection-preserving transformation $\alpha$ that fills the diagram in $\mathcal{G} r b^{\nabla}\left(G^{3}\right)$. Thus, by Lemma 5.1 .2 there is a unique connection-preserving transformation $\alpha$ making $\left(\mathcal{G}, \rho_{\kappa}, \mathcal{M}, \alpha\right)$ a multiplicative bundle gerbe with connection; this defines the functor $\mathscr{M} \mathscr{R}^{\nabla}$ on the level of objects. On the level of morphisms, one similarly composes a commutative diagram from an isomorphism in $\mathcal{F} u s \mathcal{E} x t^{\nabla}(L G)$, and then Lemma 5.1.3 implies that it yields a morphism between multiplicative bundle gerbes with connection.

Theorem 5.3.1. For a connected Lie group $G$, the two functors $\mathscr{M} \mathscr{T}^{\nabla}$ and $\mathscr{M R}^{\nabla}$ form an equivalence of categories:

$$
\mathrm{h}_{1} \mathcal{M} u l t \mathcal{G} r b^{\nabla}(G) \cong \mathcal{F} u s \mathcal{E} x t^{\nabla}(L G)
$$

Proof. We consider first the composite $\mathscr{M} \mathscr{T}^{\nabla} \circ \mathscr{M} \mathscr{R} \nabla$. Let $\mathcal{L}$ be a central extension of $L G$ equipped with a fusion product $\lambda$, a fusive superficial connection, and a multiplicative, contractible path splitting $\kappa$ of its error 1-form $\epsilon$. We denote by $\left(\mathcal{G}, \rho_{\kappa}, \mathcal{M}, \alpha\right)$ the regressed multiplicative bundle gerbe over $G$. Let $\varphi: \mathscr{T}^{\nabla} \circ \mathscr{R}^{\nabla} \longrightarrow \operatorname{id}_{\mathcal{F} u s \mathcal{B} u n^{\nabla s f}(L G)}$ be the natural transformation that establishes one half of the 
fact that $\mathscr{T}^{\nabla}$ and $\mathscr{R}^{\nabla}$ form an equivalence of categories. Thus, we have a connection-preserving, fusion-preserving isomorphism $\varphi_{\mathcal{L}}: \mathscr{T}_{\mathcal{G}}^{\nabla} \rightarrow \mathcal{L}$ over $L G$. The diagram

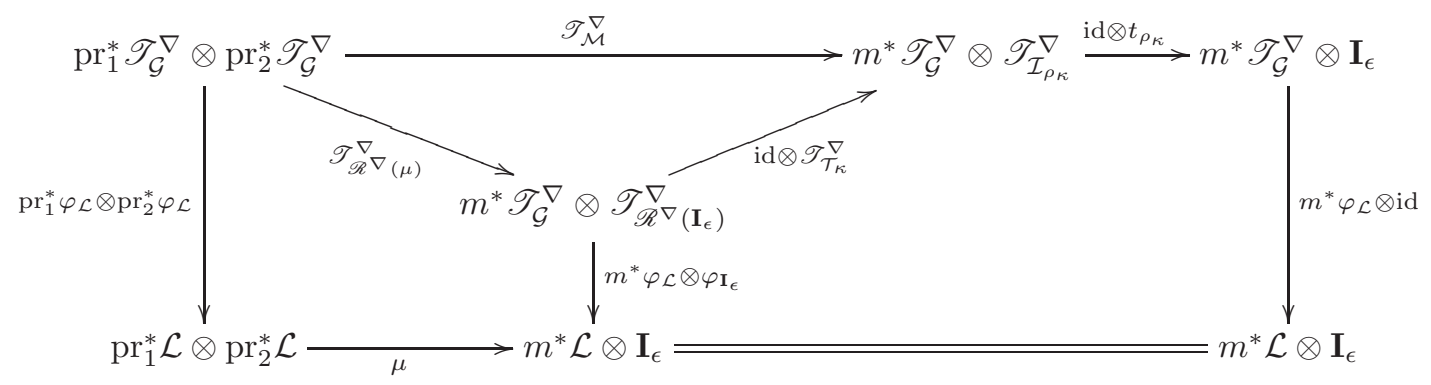

of bundle isomorphisms over $L G \times L G$ is commutative: the triangular diagram is the definition of the isomorphism $\mathcal{M}$, the left part commutes because $\varphi$ is natural, and the right part commutes due to Proposition A.4 (this uses that $\kappa$ is contractible). The bottom line is the multiplication of $\mathcal{L}$, and the top line is by definition the group structure on $\mathscr{T}_{\mathcal{G}}$. Hence, $\varphi_{\mathcal{L}}$ is connection-preserving, fusion-preserving and a group homomorphism.

Now we look at $\mathscr{M} \mathscr{R}^{\nabla} \circ \mathscr{M} \mathscr{T}^{\nabla}$. Let $(\mathcal{G}, \rho, \mathcal{M}, \alpha)$ be a multiplicative bundle gerbe with connection over $G$. Let $\mathcal{L}$ be its transgression, with fusion product $\lambda$, multiplication isomorphism $\mu$, error 1 -form $\epsilon=\tau_{S^{1}}(\rho)$ and path splitting $\kappa=\left.\tau_{[0,1]}(\rho)\right|_{P(G \times G)}$. Let $\mathcal{A}: \mathscr{R}^{\nabla} \circ \mathscr{T}^{\nabla} \longrightarrow \operatorname{id}_{\mathcal{G} r b \nabla(G)}$ be the natural transformation that establishes the second half of the fact that $\mathscr{T}^{\nabla}$ and $\mathscr{R} \nabla$ form an equivalence; thus, $\mathcal{A}_{\mathcal{G}}: \mathscr{R}^{\nabla}(\mathcal{L}) \longrightarrow \mathcal{G}$ is a connection-preserving isomorphism. The diagram

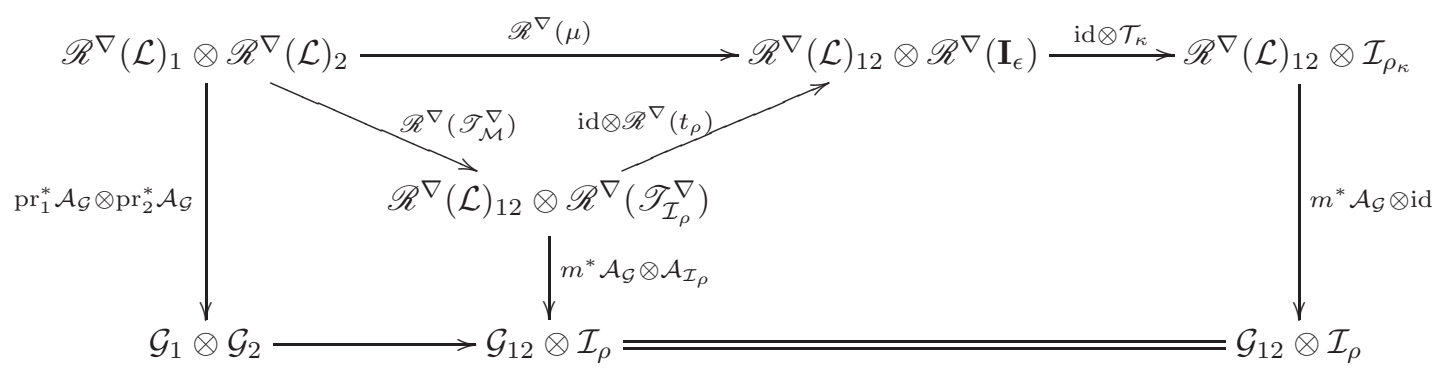

in the category $\mathrm{h}_{1} \mathcal{G} r b^{\nabla}(G \times G)$ is commutative: the triangular diagram is the definition of the multiplication $\mu$, the left part is the naturality of $\mathcal{A}$, and the right part is Proposition A.3. This means that there exists a connection-preserving transformation $\beta$ that fills the diagram. Now we are in the situation of Lemma 5.1.3, saying that $\beta$ satisfies the compatibility condition with respect to $\alpha$ and $\alpha^{\prime}$, and it becomes an isomorphism between multiplicative bundle gerbes with connection over $G$.

In the commutative diagram of Proposition 5.2.3 the maps $\mathrm{h}_{0} \mathscr{M} \mathscr{T}^{\nabla}$ and $\mathrm{h}_{0} t h$ are surjective (by Theorem 5.3.1 and by definition, respectively), hence the map

$$
\mathscr{M T}: \mathrm{h}_{0} \mathcal{M u l t \mathcal { G r }} b^{\infty}(G) \rightarrow \mathrm{h}_{0} \mathcal{F} \text { usext }{ }^{t h}(L G)
$$

is surjective. Thus, we have the next part of Theorem $\mathrm{A}$

Corollary 5.3.2. If $G$ is connected, then every thin fusion extension of LG is transgressive. 
In order to complete the proof of Theorem $\mathrm{A}$, we have to show that the map $\mathscr{M} \mathscr{T}$ is injective and so establishes a bijection.

Proposition 5.3.3. There exists a unique map $\mathscr{M} \mathscr{R}: \mathrm{h}_{0} \mathcal{F}$ susext ${ }^{t h}(L G) \rightarrow \mathrm{h}_{0} \mathcal{M} u l t \mathcal{G} r b^{\infty}(G)$ such that the diagram

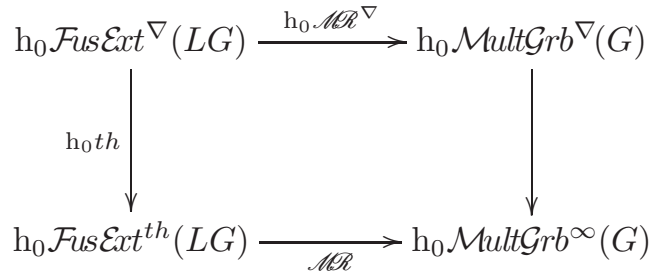

is commutative.

Proof. Uniqueness of the map follows since $\mathrm{h}_{0} t h$ is surjective. In order to define the map $\mathscr{M} \mathscr{R}$, we infer that the regression functor $\mathscr{R}^{\nabla}$ of (5.3.1) covers a functor $\mathscr{R}_{x}: \mathcal{F} u s \mathcal{B} u n(L X) \rightarrow \mathrm{h}_{1} \mathcal{G r b}(X)$ on the level without connections [Walb, Section 5.1]. Using only the group structure and the multiplicativity of the fusion product, the bundle gerbe $\mathscr{R}(\mathcal{L})$ can be equipped with a so-called strictly multiplicative structure, which in turns induces a multiplicative structure, see Sections 2 and 5 of Wal12a. It remains to prove that the diagram is commutative. We assume that we have a fusive superficial connection $\nu$ on $\mathcal{L}$ together with a multiplicative path splitting $\kappa$.

By construction, $\mathscr{M} \mathscr{R} \nabla(\mathcal{L})$ and $\mathscr{M} \mathscr{R}(\operatorname{th}(\mathcal{L})$ ) have the same underlying bundle gerbe $\mathcal{G}=\mathscr{R}(\mathcal{L})$, and the same underlying isomorphism $\mathcal{M}=\mathscr{R}(\mu)$. We show that the associators coincide; in order to do so, we prove that the isomorphism $\mathcal{M}$ and the associator $\alpha$ obtained from the strictly multiplicative structure are connection-preserving; thus, by Lemma $5.1 .2 \alpha$ equals the associator of $\mathscr{M}^{\nabla}(\mathcal{L})$.

The isomorphism $\mathcal{M}$ is induced from the map $r: Y_{1,2} \rightarrow Y_{12}$ between the surjective submersions $Y_{1,2}:=P_{1} G \times P_{1} G$ of $\mathcal{G}_{1} \otimes \mathcal{G}_{2}$ and $Y_{12}:=G \times P_{1} G$ of $\mathcal{G}_{12}$, and a lift $R: P_{1,2} \longrightarrow P_{12}$ of the map $r \times r$ to the total spaces of the principal U(1)-bundles of these gerbes. Explicitly, $r\left(\gamma_{1}, \gamma_{2}\right):=$ $\left(\gamma_{1}(1), \gamma_{1} \gamma_{2}\right)$, the bundles are $\left.P_{1,2}\right|_{\left.\left(\gamma_{1}, \gamma_{2}\right),\left(\gamma_{1}^{\prime}, \gamma_{2}^{\prime}\right)\right)}=\mathcal{L}_{\gamma_{1} \cup \gamma_{1}^{\prime}} \otimes \mathcal{L}_{\gamma_{2} \cup \gamma_{2}^{\prime}}$ and $\left.P_{12}\right|_{(g, \gamma),\left(g^{\prime}, \gamma^{\prime}\right)}=\mathcal{L}_{\gamma \cup \gamma^{\prime}}$, and $R$ is multiplication. An isomorphism induced from maps $(r, R)$ is connection-preserving with respect to the induced connections $\nu_{1,2}$ on $P_{1,2}$ and $\nu_{12}$ on $P_{12}$, if there exists a 1-form $\kappa \in \Omega^{1}\left(Y_{1,2}\right)$ such that $R^{*} \nu_{12}+\operatorname{pr}_{2}^{*} \kappa-\operatorname{pr}_{1}^{*} \kappa=\nu_{1,2}$ over $Y_{1,2} \times_{G} Y_{1,2}$. This is exactly the property of the path splitting $\kappa$; hence $\mathcal{M}$ is connection-preserving. The condition that $\kappa$ is multiplicative then implies that $\alpha$ is connection-preserving.

Now, we have two maps $\mathscr{M}$ and $\mathscr{M} \mathscr{R}$ on the bottom of the commutative diagrams of Propositions 5.2 .3 and 5.3 .3 covered along surjective maps by maps $\mathrm{h}_{0} \mathscr{M} \mathscr{T}^{\nabla}$ and $\mathrm{h}_{0} \mathscr{M} \mathscr{R} \nabla$ that are inverses of each other according to Theorem 5.3.1. This suffices to show the last part of Theorem A

Corollary 5.3.4. The maps $\mathscr{M}$ and $\mathscr{M}$ are inverses of each other, and establish a bijection

$$
\mathrm{h}_{0} \mathcal{M u l t} \mathcal{G} r b^{\infty}(G) \cong \mathrm{h}_{0} \mathcal{F} u s \mathcal{E} x t^{t h}(L G) .
$$

\section{Segal-Witten reciprocity}

Let $G$ be a Lie group and $\mathcal{L}$ be a central extension of $L G$. Let $\Sigma$ be a compact oriented surface with boundary components $b_{1}, \ldots, b_{k} \subseteq \partial \Sigma$ parameterized by orientation-preserving diffeomorphisms 
$f_{i}: S^{1} \longrightarrow b_{i}$. We have induced maps $r_{i}: C^{\infty}(\Sigma, G) \rightarrow L G$ defined by $r_{i}(\phi):=\phi \circ f_{i}$. We let $\mathcal{L}_{\Sigma}$ denote the Baer sum of the central extensions $r_{i}^{*} \mathcal{L}$ of $C^{\infty}(\Sigma, G)$,

$$
\mathcal{L}_{\Sigma}:=r_{1}^{*} \mathcal{L} \otimes \ldots \otimes r_{k}^{*} \mathcal{L} .
$$

If $\Sigma$ is obtained from two surfaces $\Sigma_{1}$ and $\Sigma_{2}$ by gluing along some boundary components, and $\rho_{i}: C^{\infty}(\Sigma, G) \rightarrow C^{\infty}\left(\Sigma_{i}, G\right)$ are the restriction maps, then we have an isomorphism

$$
\rho: \mathcal{L}_{\Sigma} \longrightarrow \rho_{1}^{*} \mathcal{L}_{\Sigma_{1}} \otimes \rho_{2}^{*} \mathcal{L}_{\Sigma_{2}}
$$

Definition 6.1. A central extension $\mathcal{L}$ of $L G$ has the smooth reciprocity property, if there exists a family $\left\{s_{\Sigma}\right\}$ of splittings $s_{\Sigma}$ of $\mathcal{L}_{\Sigma}$ for every compact oriented surface $\Sigma$, satisfying the gluing law

$$
\rho \circ s_{\Sigma}=\rho_{1}^{*} s_{\Sigma_{1}} \otimes \rho_{2}^{*} s_{\Sigma_{2}}
$$

whenever $\Sigma$ is obtained from two surfaces $\Sigma_{1}$ and $\Sigma_{2}$ by gluing along some boundary components.

By splitting we mean a smooth map $s_{\Sigma}: C^{\infty}(\Sigma, G) \rightarrow \mathcal{L}_{\Sigma}$ such that $p \circ s_{\Sigma}=\mathrm{id}_{C^{\infty}(\Sigma, G)}$, where $p$ is the projection $p: \mathcal{L}_{\Sigma} \longrightarrow C^{\infty}(\Sigma, G)$.

\section{Remark 6.2.}

(i) Above definition is derived from a definition due to Brylinski and McLaughlin [BM94] and a gluing law from [BM96]; in these references the definition is attributed to Segal [Seg04.

(ii) There is a complex version of the reciprocity property, where $G$ is a complex Lie group, $\Sigma$ is a Riemann surface, and $C^{\infty}(\Sigma, G)$ is replaced by $\operatorname{Hol}(\Sigma, G)$, the holomorphic maps from $\Sigma$ to $G$ BM96.

(iii) The smooth reciprocity property is a property of the underlying principal $\mathrm{U}(1)$-bundle of $\mathcal{L}$, i.e. the group structure is neglected. For the complex reciprocity property one additionally assumes that the sections $s_{\Sigma}$ are group homomorphisms.

The Segal-Witten reciprocity law states that every transgressive central extension of the loop group of a complex Lie group has the complex reciprocity property. The following result is a weaker version adapted to the smooth reciprocity property.

Theorem 6.3. Every transgressive central extension of the loop group of any Lie group has the smooth reciprocity property.

Proof. Let $(\mathcal{G}, \rho, \mathcal{M}, \alpha)$ be a multiplicative bundle gerbe with connection over $G$, and let $\mathcal{L}$ be the corresponding central extension. Suppose $\Sigma$ is a compact oriented surface, $\phi: \Sigma \rightarrow G$ is a smooth map, and $\mathcal{T}_{i}$ is a trivialization of $\left.\phi^{*} \mathcal{G}\right|_{b_{i}}$ for every boundary component $b_{i}$. The surface holonomy $\mathcal{A}_{\Sigma}\left(\phi, \mathcal{T}_{1}, \ldots, \mathcal{T}_{k}\right) \in \mathrm{U}(1)$ of $\mathcal{G}$ with boundary conditions $\mathcal{T}_{1}, \ldots, \mathcal{T}_{k}$ is defined in the following way. Choose a trivialization $\mathcal{S}: \phi^{*} \mathcal{G} \longrightarrow \mathcal{I}_{\omega}$. For each boundary component, we have two trivializations that differ by a $\mathrm{U}(1)$-bundle $T_{i}$ with connection over $b_{i}$, i.e. $\left.\mathcal{S}\right|_{b_{i}} \cong \mathcal{T}_{i} \otimes T_{i}$ Then,

$$
\mathcal{A}_{\Sigma}\left(\phi, \mathcal{T}_{1}, \ldots, \mathcal{T}_{k}\right):=\exp \left(\int_{\Sigma} \omega\right) \cdot \prod_{i=1}^{k} \operatorname{Hol}_{T_{i}}\left(b_{i}\right)^{-1}
$$


With boundary parameterizations $f_{i}: S^{1} \longrightarrow b_{i}$ we have $f_{i}^{*} \mathcal{T}_{i} \in\left(r_{i}^{*} \mathcal{L}\right)_{\phi}$ and so $\left.\left(f_{1}^{*} \mathcal{T}_{1}, \ldots, f_{k}^{*} \mathcal{T}_{k}\right) \in \mathcal{L}_{\Sigma}\right|_{\phi}$. Now, a splitting $s_{\Sigma}: C^{\infty}(\Sigma, G) \rightarrow \mathcal{L}_{\Sigma}$ is defined by

$$
s_{\Sigma}(\phi)=\left(f_{1}^{*} \mathcal{T}_{1}, \ldots, f_{k}^{*} \mathcal{T}_{k}\right) \cdot \mathcal{A}_{\Sigma}\left(\phi, \mathcal{T}_{1}, \ldots, \mathcal{T}_{k}\right) .
$$

This splitting satisfies the gluing property since surface holonomy has a more general gluing property, see [CJM02, Proposition 3.1] and Walb, Lemma 3.3.3 (c)].

\section{Remark 6.4.}

(i) Theorem 6.3 is proved in Bry93, Theorem 6.2.1] and [BM94, Theorem 5.9] for simply-connected Lie groups, and in the latter reference it is claimed that it generalizes to arbitrary Lie groups by a (left out) computation in simplicial Deligne cohomology.

(ii) By Theorem $\mathrm{A}$ the transgressive central extensions are precisely the thin fusion extension. For a given thin fusion extension $\mathcal{L}$ the splitting $s_{\Sigma}$ can be constructed directly from a choice of a fusive superficial connection $\nu$ that integrates the given thin structure. Indeed, the surface holonomy $\mathcal{A}_{\Sigma}\left(\mathcal{T}_{1}, \ldots, \mathcal{T}_{k}\right)$ can be defined directly from $\nu$ and the fusion product, see [Walb, Section 5.3].

It is an interesting question whether or not the sections $s_{\Sigma}$ can be chosen multiplicative, i.e. to be group homomorphisms. In BM94 it is claimed that this is possible for transgressive central extensions of loop groups of arbitrary Lie groups. In Bry] on pages 2 and 21 Brylinski withdraws that statement, and claims that only the sections for the complex reciprocity property can be chosen multiplicative. Based on this claim, it is proved in BM96 that the complex reciprocity property characterizes transgressive central extensions of the loop group of a connected semisimple complex Lie group.

In the present paper we make no claims about complex Lie groups. In the following we only show via examples that there exist transgressive central extensions for which the sections $s_{\Sigma}$ cannot be chosen multiplicative (Example 6.6), and that there exist central extensions that have the smooth reciprocity property but are not transgressive (Example 6.7). For preparation, we need the following.

Proposition 6.5. The splitting $s_{\Sigma}$ constructed in Theorem 6.3 satisfies

$$
s_{\Sigma}\left(\phi_{1} \phi_{2}\right)=s_{\Sigma}\left(\phi_{1}\right) \cdot s_{\Sigma}\left(\phi_{2}\right) \cdot \exp 2 \pi \mathrm{i}\left(-\int_{\Sigma}\left(\phi_{1}, \phi_{2}\right)^{*} \rho\right)
$$

where $\rho \in \Omega^{2}(G \times G)$ is the 2-form of the multiplicative gerbe.

Proof. Suppose $\phi_{1}, \phi_{2}: \Sigma \longrightarrow G$. We may have chosen trivializations $\mathcal{S}_{j}: \phi_{j}^{*} \mathcal{G} \rightarrow \mathcal{I}_{\omega_{j}}$. Then, we get another trivialization $\mathcal{S}$ defined by

$$
\left(\phi_{1} \phi_{2}\right)^{*} \mathcal{G} \stackrel{\left(\phi_{1}, \phi_{2}\right)^{*} \mathcal{M}^{-1}}{\longrightarrow} \phi_{1}^{*} \mathcal{G} \otimes \phi_{2}^{*} \mathcal{G} \otimes \mathcal{I}_{-\left(\phi_{1}, \phi_{2}\right)^{*} \rho} \stackrel{\mathcal{S}_{1} \otimes \mathcal{S}_{2}}{\longrightarrow} \mathcal{I}_{\omega_{1}+\omega_{2}-\left(\phi_{1}, \phi_{2}\right)^{*} \rho}
$$

We further have trivializations $\mathcal{T}_{i j}: \phi_{j}^{*} \mathcal{G}_{b_{i}} \rightarrow \mathcal{I}_{0}$ for $j=1,2$ and $i=1, \ldots, k$ and the difference bundles $\left.\mathcal{S}_{j}\right|_{b_{i}} \cong \mathcal{T}_{i j} \otimes T_{i j}$. We consider the trivialization $\mathcal{T}_{i}$ defined by

$$
\left.\left.\left.\left(\phi_{1} \phi_{2}\right)^{*} \mathcal{G}\right|_{b_{i}} \stackrel{\left(\phi_{1}, \phi_{2}\right)^{*} \mathcal{M}^{-1}}{\longrightarrow} \phi_{1}^{*} \mathcal{G}\right|_{b_{i}} \otimes \phi_{2}^{*} \mathcal{G}\right|_{b_{i}} \stackrel{\mathcal{T}_{i 1} \otimes \mathcal{T}_{i 2}}{\longrightarrow} \mathcal{I}_{0}
$$

and obtain

$$
\left.\mathcal{S}\right|_{b_{i}}=\left.\left.\left(\left.\left.\mathcal{S}_{1}\right|_{b_{i}} \otimes \mathcal{S}_{2}\right|_{b_{i}}\right) \circ\left(\phi_{1}, \phi_{2}\right)^{*} \mathcal{M}^{-1}\right|_{b_{i}} \cong\left(\mathcal{T}_{i 1} \otimes \mathcal{T}_{i 2}\right) \circ\left(\phi_{1}, \phi_{2}\right)^{*} \mathcal{M}^{-1}\right|_{b_{i}} \otimes T_{i 1} \otimes T_{i 2}=\mathcal{T}_{i} \otimes T_{i 1} \otimes T_{i 2} .
$$


Thus,

$$
\begin{aligned}
\mathcal{A}_{\Sigma}\left(\phi_{1} \phi_{2}, \mathcal{T}_{1}, \ldots, \mathcal{T}_{k}\right)= & \exp 2 \pi \mathrm{i}\left(\int_{\Sigma} \omega_{1}+\omega_{2}-\left(\phi_{1}, \phi_{2}\right)^{*} \rho\right) \cdot \prod_{i=1}^{k} \operatorname{Hol}_{T_{i 1} \otimes T_{i 2}}\left(b_{i}\right)^{-1} \\
& =\mathcal{A}_{\Sigma}\left(\phi_{1}, \mathcal{T}_{11}, \ldots, \mathcal{T}_{k 1}\right) \cdot \mathcal{A}_{\Sigma}\left(\phi_{1}, \mathcal{T}_{12}, \ldots, \mathcal{T}_{k 2}\right) \cdot \exp 2 \pi \mathrm{i}\left(\int_{\Sigma}-\left(\phi_{1}, \phi_{2}\right)^{*} \rho\right) .
\end{aligned}
$$

The product of $f_{i}^{*} \mathcal{T}_{i 1} \in \mathcal{L}_{r_{i}\left(\phi_{1}\right)}$ and $f_{i}^{*} \mathcal{T}_{i 2} \in \mathcal{L}_{r_{i}\left(\phi_{2}\right)}$ is $f_{i}^{*} \mathcal{T}_{i}$. The claim follows by computing $s_{\Sigma}\left(\phi_{1} \phi_{2}\right)$ using (6.1).

The meaning of the calculation of Proposition 6.5 is that

$$
\eta: C^{\infty}(\Sigma, G) \times C^{\infty}(\Sigma, G) \rightarrow \mathrm{U}(1):\left(\phi_{1}, \phi_{2}\right) \mapsto \exp 2 \pi \mathrm{i}\left(\int_{\Sigma}\left(\phi_{1}, \phi_{2}\right)^{*} \rho\right)
$$

is a classifying 2-cocycle for the (topologically trivializable) central extension $\mathcal{L}_{\Sigma}$. Note that $\eta$ is a coboundary if and only if $\mathcal{L}_{\Sigma}$ has a multiplicative section. Also note that if $G$ is abelian and $\eta$ is a coboundary, then $\eta$ is symmetric.

Example 6.6. Consider $G=\mathrm{U}(1)$ and the central extension $\mathcal{L}=\mathcal{L}_{P}=\mathcal{L}_{\mathbb{Z}}(-1,0,1)$, i.e. the transgression of the trivial gerbe with multiplicative structure given by the Poincaré bundle $P$ over $T=\mathrm{U}(1) \times \mathrm{U}(1)$, see Examples 2.5 and 4.12 By Theorem $6.3 \mathcal{L}$ has the smooth reciprocity property. Here, $\rho=\operatorname{pr}_{1}^{*} \theta \wedge \operatorname{pr}_{2}^{*} \theta \in \Omega^{2}(T)$. It is easy to see that we have $s^{*} \rho=-\rho$, where $s: T \longrightarrow T:\left(z, z^{\prime}\right) \longmapsto\left(z^{\prime}, z\right)$. This shows that $\eta$ is skew-symmetric. But $\eta$ can only be symmetric and skew-symmetric if $\eta=1$. In order to see that this is not the case, we note that

$$
\int_{T} \rho=1
$$

Then, there must be an embedding $\phi: D^{2} \longrightarrow T$ of a disc such that $\int \phi^{*} \rho \notin \mathbb{Z}$. Defining $\phi_{1}, \phi_{2}$ by composing with the projections $\operatorname{pr}_{1}, \operatorname{pr}_{2}: T \rightarrow \mathrm{U}(1)$, we get $\eta\left(\phi_{1}, \phi_{2}\right) \neq 0$. This shows that $\eta$ is not symmetric, so that $\mathcal{L}_{D^{2}}$ has no multiplicative section. Hence, $\mathcal{L}$ is a transgressive central extension that has the smooth reciprocity property but does not admit multiplicative sections.

Example 6.7. We let $G=\mathrm{U}(1)$ and $\mathcal{L}_{\mathbb{R}}(\gamma)$ be the basic central extension of $L \mathrm{U}(1)$ constructed in Example 4.12 depending on $\gamma \in \mathbb{R}$. It is clear that $\mathcal{L}_{\mathbb{R}}(\gamma)$ has the smooth reciprocity property, because it is topologically trivial. On the other hand, we have seen in Example 4.12 that it cannot be equipped with the structure of a thin fusion extension unless $\gamma \in \mathbb{Z}$. By Proposition 5.2.2 it is hence not transgressive. In other words, the smooth reciprocity property is not sufficient to characterize transgressive central extensions.

The conclusion of this section is that the reciprocity property (in its original form or in the version of Definition 6.1) does not properly characterize transgressive central extensions of non-complex Lie groups. The theory of loop fusion and thin homotopy equivariance that we have developed in this article provides such characterization, valid for all connected Lie groups.

\section{A Regression of trivial fusion bundles}

In this appendix we discuss the regression of trivial bundles with trivial fusion products (but non-trivial connections) over the loop space $L X$ of a connected smooth manifold $X$. For this purpose, we restrict 
the constructions of [Walb, Sections 5 and 6 ] to that case; this has not yet been worked out explicitly.

For $x \in X$ we consider the diffeological space $P_{x} X$ of paths in $X$ starting at $x$ with sitting instants, equipped with the subduction $\mathrm{ev}_{1}: P_{x} X \rightarrow X: \gamma \mapsto \gamma(1)$ (the diffeological analog of a surjective submersion). Two paths with the same end point compose to a loop via the smooth map $\cup_{x}: P_{x} X^{[2]} \longrightarrow L X:\left(\gamma_{1}, \gamma_{2}\right) \mapsto \overline{\gamma_{2}} \star \gamma_{1}$.

Suppose $\epsilon \in \Omega^{1}(L X)$ is a superficial connection on the trivial bundle $\mathbf{I}$ that is fusive with respect to the trivial fusion product. The regression $\mathscr{R}_{x}^{\nabla}\left(\mathbf{I}_{\epsilon}\right)$ is a bundle gerbe with connection over $X$, composed of the subduction $\mathrm{ev}_{1}: P_{x} X \rightarrow X$, the principal $S^{1}$-bundle with connection $\cup_{x}^{*} \mathbf{I}_{\epsilon}$, and the identity bundle gerbe product, which is connection-preserving because $\epsilon$ is fusive. The difficult part is to specify a curving: a 2-form $B_{\epsilon} \in \Omega^{2}\left(P_{x} X\right)$ such that $\operatorname{pr}_{2}^{*} B_{\epsilon}-\operatorname{pr}_{1}^{*} B_{\epsilon}=\operatorname{curv}\left(\cup_{x}^{*} \mathbf{I}_{\epsilon}\right)=\cup_{x}^{*} \mathrm{~d} \epsilon$.

Such a curving can be constructed because $\epsilon$ is superficial, see Walb, Section 5.2]. The construction uses a bijection between the 2 -forms on a diffeological space $Y$ and certain smooth maps $\mathcal{B} Y \rightarrow \mathrm{U}(1)$ on the space $\mathcal{B} Y$ of bigons in $Y$. A bigon is a smooth fixed-ends homotopy $\Sigma$ between two paths in $Y$, and the correspondence between a smooth map $G: \mathcal{B} Y \rightarrow \mathrm{U}(1)$ and a 2 -form $B \in \Omega^{2}(Y)$ is established by the relation

$$
G(\Sigma)=\exp 2 \pi \mathrm{i}\left(-\int_{\Sigma} B\right)
$$

Suppose $\Sigma \in \mathcal{B} P_{x} X$ is a bigon between a path $\gamma_{0} \in P P_{x} X$ and a path $\gamma_{1} \in P P_{x} X$. Thus, it is a smooth map $\Sigma:[0,1]^{2} \rightarrow P_{x} X$ such that $\Sigma(0, t)=\gamma_{0}(t)$ and $\Sigma(1, t)=\gamma_{1}(t)$. For each $t \in[0,1]$ we
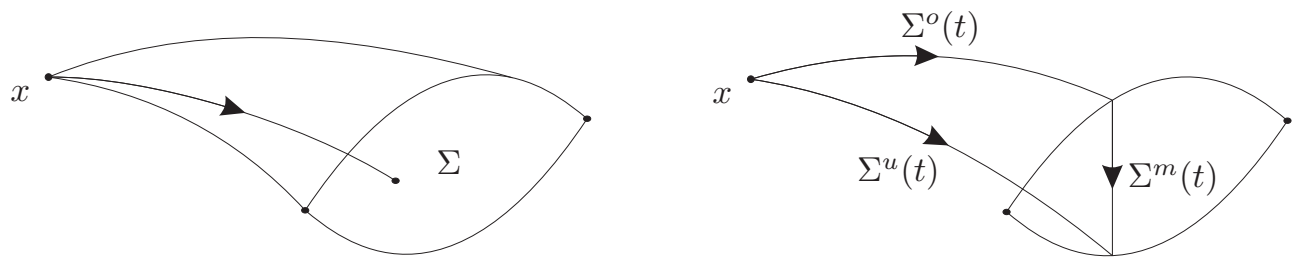

Figure 3: The picture on the left shows a bigon $\Sigma$ in $P_{x} X$ : it can be regarded as a bigon in $X$ that has for each of its points a chosen path connecting $x$ with that point. The picture on the right shows the three paths associated to a bigon $\Sigma$ and $t \in[0,1]$.

extract a loop $\gamma_{\Sigma}(t) \in L X$ defined by

$$
\gamma_{\Sigma}(t):=\left(\Sigma^{m}(t) \star \Sigma^{o}(t)\right) \cup\left(\mathrm{id} \star \Sigma^{u}(t)\right)
$$

where $\Sigma^{m}(t), \Sigma^{o}(t)$, and $\Sigma^{u}(t)$ are the three paths depicted in Figure 3. Thus, $\gamma_{\Sigma}$ is a path in $L X$ that starts and ends at flat loops.

Using a smoothing function $\phi:[0,1] \longrightarrow[0,1]$, we define a parameterized version $\Sigma_{\sigma}$ for $\sigma \in[0,1]$ by $\Sigma_{\sigma}(s, t):=\Sigma(\phi(s) \sigma, t)$, i.e. $\Sigma_{0}$ is the identity bigon at the path $\gamma_{0}$, and $\Sigma_{1}=\Sigma$. Now we consider $h:[0,1]^{2} \rightarrow L X:(\sigma, t) \mapsto \gamma_{\Sigma_{\sigma}}(t)$. By Walb, Lemma 5.2.1] the curving $B_{\epsilon}$ corresponds to the smooth map

$$
G_{\epsilon}: \mathcal{B} P_{x} X \rightarrow \mathrm{U}(1): \Sigma \longmapsto \exp 2 \pi \mathrm{i}\left(-\int_{[0,1]^{2}} h^{*} \operatorname{curv}\left(\mathbf{I}_{\epsilon}\right)\right) .
$$

As $\operatorname{curv}\left(\mathbf{I}_{\epsilon}\right)=\mathrm{d} \epsilon$ we want to apply Stokes' theorem. Along the boundary of $[0,1]^{2}$, the map $h$ is as follows: $h(0, t)=\gamma_{0}(t) \cup \gamma_{0}(t), h(1, t)=\gamma_{\Sigma}(t), h(\sigma, 0)=\gamma_{0}(0) \cup \gamma_{0}(0)$, and $h(\sigma, 1)=\gamma_{0}(1) \cup \gamma_{0}(1)$. As 
$\epsilon$ is fusive, we have $b^{*} \epsilon=0$, where $b: P X \rightarrow L X$ is the inclusion of flat loops, see Section 3.1. Thus, we get

$$
G_{\epsilon}(\Sigma)=\exp 2 \pi \mathrm{i}\left(-\int_{0}^{1} \gamma_{\Sigma}^{*} \epsilon\right)
$$

The regressed bundle gerbe $\mathscr{R}_{x}^{\nabla}\left(\mathbf{I}_{\epsilon}\right)$ is not the trivial bundle gerbe (that one would have the identity subduction $\mathrm{id}_{X}$ ). It is trivializable, but not canonically trivializable. However, a trivialization $\mathcal{T}_{\kappa}$ can be obtained from a path splitting $\kappa \in \Omega^{1}(P X)$ of $\epsilon$, see Definition 4.4 The trivialization $\mathcal{T}_{\kappa}$ is composed of the principal $\mathrm{U}(1)$-bundle $\mathbf{I}_{-\kappa}$ over $P_{x} X$ and of the bundle isomorphism

$$
\text { id }: \cup_{x}^{*} \mathbf{I}_{\epsilon} \otimes \operatorname{pr}_{2}^{*} \mathbf{I}_{-\kappa} \longrightarrow \operatorname{pr}_{1}^{*} \mathbf{I}_{-\kappa}
$$

over $P_{x} X^{[2]}$, which is connection-preserving due to the defining property of a path splitting. There exists a unique 2-form $\rho_{\kappa} \in \Omega^{2}(X)$ such that $\mathcal{T}_{\kappa}: \mathscr{R}_{x}^{\nabla}\left(\mathbf{I}_{\epsilon}\right) \rightarrow \mathcal{I}_{\rho_{\kappa}}$ is a connection-preserving isomorphism; this 2-form is characterized by the condition $\operatorname{ev}_{1}^{*} \rho_{\kappa}=B_{\epsilon}-\mathrm{d} \kappa$.

Lemma A.1. Suppose $\rho \in \Omega^{2}(X)$ and $\epsilon:=\tau_{S^{1}}(\rho) \in \Omega^{1}(L X)$. Then, $\kappa:=\tau_{[0,1]}(\rho)$ is a path splitting for $\epsilon$, and $B_{\epsilon}=\mathrm{ev}_{1}^{*} \rho+\mathrm{d} \kappa$. In particular, $\rho_{\kappa}=\rho$.

Proof. That $\kappa$ is a path splitting for $\epsilon$ has been checked in Example 4.5. Let $\Sigma$ be a bigon in $P_{x} X$ between a path $\gamma_{0}$ and a path $\gamma_{1}$. We have

$$
G_{\epsilon}(\Sigma)=\exp 2 \pi \mathrm{i}\left(-\int_{0}^{1} \gamma_{\Sigma}^{*} \epsilon\right)=\exp 2 \pi \mathrm{i}\left(\int_{[0,1] \times S^{1}} h_{\gamma_{\Sigma}}^{*} \rho\right)
$$

with $h_{\gamma_{\Sigma}}:[0,1] \times S^{1} \rightarrow X$ defined by $h_{\gamma_{\Sigma}}(t, z):=\gamma_{\Sigma}(t)(z)$. We obtain from the definition A.2 of $\gamma_{\Sigma}$ :

$$
h_{\gamma_{\Sigma}}(t, z)= \begin{cases}\gamma_{0}(t)(4 z) & \text { if } 0 \leq z \leq \frac{1}{4} \\ \operatorname{ev}_{1}(\Sigma(4 z-1)(t)) & \text { if } \frac{1}{4} \leq z \leq \frac{1}{2} \\ \gamma_{1}(t)(3-4 z) & \text { if } \frac{1}{2} \leq z \leq \frac{3}{4} \\ x & \text { if } \frac{3}{4} \leq z \leq 1\end{cases}
$$

Splitting the domain of integration into those four parts and taking care with the involved orientations yields

$$
\exp 2 \pi \mathrm{i}\left(\int_{[0,1] \times S^{1}} h_{\gamma_{\Sigma}}^{*} \rho\right)=\exp 2 \pi \mathrm{i}\left(-\int_{\Sigma} \mathrm{ev}_{1}^{*} \rho+\int_{\gamma_{0}} \kappa-\int_{\gamma_{1}} \kappa\right) .
$$

Finally, Stokes' theorem gives

$$
\int_{\Sigma} \mathrm{d} \kappa=-\int_{\gamma_{0}} \kappa+\int_{\gamma_{1}} \kappa
$$

All together, we obtain

$$
G_{\epsilon}(\Sigma)=\exp 2 \pi \mathrm{i}\left(-\int_{\Sigma}\left(\mathrm{ev}_{1}^{*} \rho+\mathrm{d} \kappa\right)\right) .
$$

Using (A.1), we get the claimed equality. 
As regression is a monoidal functor, we want to make sure that the trivialization $\mathcal{T}_{\kappa}$ is compatible with that monoidal structure.

Lemma A.2. Suppose $\epsilon_{1}, \epsilon_{2}$ are superficial connections on the trivial bundle $\mathbf{I}$ over $L X$, and fusive with respect to the trivial fusion product. Suppose $\kappa_{1}$ and $\kappa_{2}$ are path splittings for $\epsilon_{1}$ and $\epsilon_{2}$, respectively. Then, we have $\rho_{\kappa_{1}+\kappa_{2}}=\rho_{\kappa_{1}}+\rho_{\kappa_{2}}$, and there exists a connection-preserving transformation

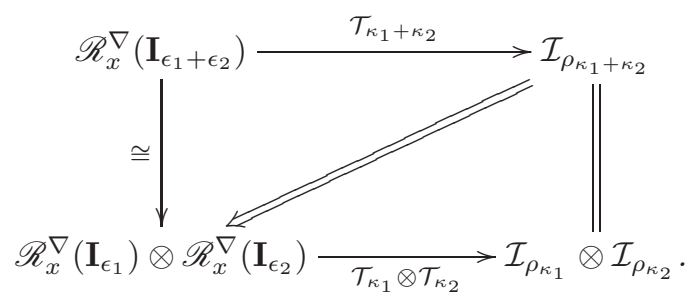

Proof. The isomorphism $\mathscr{R}_{x}^{\nabla}\left(\mathbf{I}_{\epsilon_{1}+\epsilon_{2}}\right) \cong \mathscr{R}_{x}^{\nabla}\left(\mathbf{I}_{\epsilon_{1}}\right) \otimes \mathscr{R}_{x}^{\nabla}\left(\mathbf{I}_{\epsilon_{2}}\right)$ that implements that $\mathscr{R}_{x}^{\nabla}$ is monoidal is induced from the connection-preserving, fusion-preserving isomorphism id: $\mathbf{I}_{\epsilon_{1}+\epsilon_{2}} \longrightarrow \mathbf{I}_{\epsilon_{1}} \otimes \mathbf{I}_{\epsilon_{2}}$. In particular, we have $B_{\epsilon_{1}+\epsilon_{2}}=B_{\epsilon_{1}}+B_{\epsilon_{2}}$. We calculate ev $v_{1}^{*}\left(\rho_{\kappa_{1}}+\rho_{\kappa_{2}}\right)=B_{\epsilon_{1}}-\mathrm{d} \kappa_{1}+B_{\epsilon_{1}}-\mathrm{d} \kappa_{1}=B_{\epsilon_{1}+\epsilon_{2}}+$ $\mathrm{d}\left(\kappa_{1}+\kappa_{2}\right)$; this shows that $\rho_{\kappa_{1}+\kappa_{2}}=\rho_{\kappa_{1}}+\rho_{\kappa_{2}}$. The announced connection-preserving transformation is now simply induced by the connection-preserving isomorphism id : $\mathbf{I}_{-\left(\kappa_{1}+\kappa_{2}\right)} \rightarrow \mathbf{I}_{-\kappa_{1}} \otimes \mathbf{I}_{-\kappa_{2}}$.

The next two propositions describe the relation between the trivialization $\mathcal{T}_{\kappa}$ of the regressed bundle gerbe $\mathscr{R}_{x}^{\nabla}\left(\mathbf{I}_{\epsilon}\right)$, the canonical trivialization $t_{\rho}$ of $\mathscr{T}_{\mathcal{I}_{\rho}}^{\nabla}$, and the two natural equivalences

$$
\mathcal{A}: \mathscr{R}_{x}^{\nabla} \circ \mathscr{T}^{\nabla} \longrightarrow \operatorname{id}_{\mathrm{h}_{1} \mathcal{G} r b \nabla(X)} \quad \text { and } \quad \varphi: \mathscr{T}^{\nabla} \circ \mathscr{R}_{x}^{\nabla} \longrightarrow \operatorname{id}_{\mathcal{F} u s \mathcal{B} u n^{\nabla s f}(X)}
$$

that establish that the functors $\mathscr{R}_{x}^{\nabla}$ and $\mathscr{T}^{\nabla}$ form an equivalence of categories Walb, Theorem A].

Proposition A.3. Suppose $\rho \in \Omega^{2}(X)$. Let $t_{\rho}: \mathscr{T}_{\mathcal{I}_{\rho}}^{\nabla} \rightarrow \mathbf{I}_{\epsilon}$ be the canonical trivialization, with $\epsilon=\tau_{S^{1}}(\rho) \in \Omega^{1}(L X)$. Let $\mathcal{A}_{\mathcal{I}_{\rho}}: \mathscr{R}_{x}^{\nabla}\left(\mathscr{T}_{\mathcal{I}_{\rho}}^{\nabla}\right) \rightarrow \mathcal{I}_{\rho}$ be the component of the natural equivalence $\mathcal{A}$ at $\mathcal{I}_{\rho}$. Let $\kappa:=\tau_{[0,1]}(\rho)$ be the canonical path splitting of $\epsilon$ and let $\mathcal{T}_{\kappa}: \mathscr{R}_{x}^{\nabla}\left(\mathbf{I}_{\epsilon}\right) \longrightarrow \mathcal{I}_{\rho}$ be the corresponding trivialization. Then, there exists a connection-preserving transformation

$$
\mathcal{A}_{\mathcal{I}_{\rho}} \cong \mathcal{T}_{\kappa} \circ \mathscr{R}_{x}^{\nabla}\left(t_{\rho}\right) \text {. }
$$

Proof. The isomorphism $\mathscr{R}_{x}^{\nabla}\left(t_{\rho}\right): \mathscr{R}_{x}^{\nabla}\left(\mathscr{T}_{\mathcal{I}_{\rho}}^{\nabla}\right) \rightarrow \mathscr{R}_{x}^{\nabla}\left(\mathbf{I}_{\epsilon}\right)$ is induced from the bundle isomorphism $\cup^{*} t_{\rho}: \cup^{*} \mathscr{T}_{\mathcal{I}_{\rho}}^{\nabla} \rightarrow \mathbf{I}_{\cup^{*} \epsilon}$ over $P_{x} X^{[2]}$. The composition $\mathcal{T}_{\kappa} \circ \mathscr{R}_{x}^{\nabla}\left(t_{\rho}\right)$ is thus given by the $S^{1}$-bundle $\mathbf{I}_{-\kappa}$ and the isomorphism

$$
\cup_{x}^{*} \mathscr{T}_{\mathcal{I}_{\rho}}^{\nabla} \otimes \operatorname{pr}_{2}^{*} \mathbf{I}_{-\kappa} \stackrel{\cup_{x}^{*} t_{\rho} \otimes \mathrm{id}}{\longrightarrow} \cup_{x}^{*} \mathbf{I}_{\epsilon} \otimes \operatorname{pr}_{2}^{*} \mathbf{I}_{-\kappa} \stackrel{\mathrm{id}}{\longrightarrow} \operatorname{pr}_{1}^{*} \mathbf{I}_{-\kappa}
$$

Next we describe the connection-preserving isomorphism $\mathcal{A}_{\mathcal{I}_{\rho}}$ following [Walb, Section 6.1]. It consists of an $S^{1}$-bundle $Q$ over $P_{x} X$ with connection, and of a connection-preserving bundle isomorphism $\alpha: \cup_{x}^{*} \mathscr{T}_{\mathcal{I}_{\rho}}^{\nabla} \otimes \operatorname{pr}_{2}^{*} Q \rightarrow \operatorname{pr}_{1}^{*} Q$ over $P_{x} X^{[2]}$

The fibre of $Q$ over $\gamma \in P_{x} X$ consists of triples $\left(\mathcal{T}, t_{0}, t\right)$, where $\mathcal{T}: \gamma^{*} \mathcal{I}_{\rho} \longrightarrow \mathcal{I}_{0}$ is a trivialization (in turn consisting of an $S^{1}$-bundle $T$ with connection over $[0,1]$ and of a connection-preserving bundle isomorphism which here is necessarily the identity $\left.\tau=\mathrm{id}_{T}\right), t_{0} \in T_{0}$ and $t \in T_{1}$. Two triples $\left(\mathcal{T}, t_{0}, t\right)$ and $\left(\mathcal{T}^{\prime}, t_{0}^{\prime}, t^{\prime}\right)$ are identified if there exists a connection-preserving transformation $\varphi: \mathcal{T} \Longrightarrow \mathcal{T}^{\prime}$ such 
that $\varphi\left(t_{0}\right)=t_{0}^{\prime}$ and $\varphi(t)=t^{\prime}$. The $S^{1}$-action on $S^{1}$ is $\left(\mathcal{T}, t_{0}, t\right) \cdot z:=\left(\mathcal{T}, t_{0}, t \cdot z\right)$. In our situation, $Q$ has a canonical section $s: P_{x} X \longrightarrow Q:(\gamma, z) \mapsto\left(\operatorname{id}_{\mathcal{I}_{0}}, 1,1\right)$, using that $\gamma^{*} \mathcal{I}_{\rho}=\mathcal{I}_{\gamma^{*} \rho}=\mathcal{I}_{0}$. The bundle isomorphism $\alpha$ is over a point $\left(\gamma_{1}, \gamma_{2}\right) \in P_{x} X^{[2]}$ a map

$$
\alpha:\left.\mathscr{T}_{\mathcal{I}_{\rho}}\right|_{\gamma_{1} \cup \gamma_{2}} \otimes Q_{\gamma_{2}} \rightarrow Q_{\gamma_{1}},
$$

and it is characterized by $\alpha\left(t_{\rho}\left(\gamma_{1} \cup \gamma_{2}\right) \otimes s\left(\gamma_{2}\right)\right)=s\left(\gamma_{1}\right)$. The connection on $Q$ is defined via its parallel transport. Using the section $s: P_{x} X \rightarrow Q$, it suffices to define a 1-form on $P_{x} X$, and we do this by defining a smooth map $F: P P_{x} X \rightarrow S^{1}$. This map is given by

$$
F(\gamma)=\int_{h_{\gamma}} \rho
$$

where $h_{\gamma}:[0,1]^{2} \rightarrow X$ is defined by $h_{\gamma}(s, t)=\gamma(s)(t)$. However, this map characterizes precisely the parallel transport of the 1 -form $-\kappa=-\tau_{[0,1]}(\rho) \in \Omega^{1}\left(P_{x} X\right)$.

Summarizing, $s$ defines a connection-preserving transformation between $\mathcal{A}_{\mathcal{I}_{0}}$ and the the trivialization consisting of the trivial bundle $\mathbf{I}_{-\kappa}$ and of the isomorphism (A.3).

Proposition A.4. Suppose $\epsilon \in \Omega^{1}(L X)$ is a superficial connection on the trivial U(1)-bundle over $L X$, and fusive with respect to the trivial fusion product. Let $\varphi_{\mathbf{I}_{\epsilon}}: \mathscr{T}_{\mathscr{R}_{x}^{\nabla}\left(\mathbf{I}_{\epsilon}\right)}^{\nabla} \rightarrow \mathbf{I}_{\epsilon}$ be the component of the natural equivalence $\varphi$ at $\mathbf{I}_{\epsilon}$. Let $\kappa \in \Omega^{1}(P X)$ be a contractible path splitting for $\epsilon$, and let $\mathcal{T}_{\kappa}: \mathscr{R}_{x}^{\nabla}\left(\mathbf{I}_{\epsilon}\right) \longrightarrow \mathcal{I}_{\rho_{\kappa}}$ be the corresponding trivialization. Let $t_{\rho_{\kappa}}: \mathscr{T}_{\mathcal{I}_{\rho_{\kappa}}}^{\nabla} \longrightarrow \mathbf{I}_{\epsilon_{\kappa}}$ be the canonical trivialization with $\epsilon_{\kappa}=\tau_{S^{1}}\left(\rho_{\kappa}\right)$. Then,

$$
\varphi_{\mathbf{I}_{\epsilon}}=t_{\rho_{\kappa}} \circ \mathscr{T}_{\mathcal{T}_{\kappa}}^{\nabla}
$$

in particular, $\epsilon_{\kappa}=\epsilon$.

Proof. Note that (A.4) is an equality between two connection-preserving bundle isomorphisms going from $\stackrel{\mathscr{T}_{\mathscr{R} \nabla}\left(\mathbf{I}_{\epsilon}\right)}{\nabla}$ to $\mathbf{I}_{\epsilon}$ and $\mathbf{I}_{\epsilon_{\kappa}}$, respectively. This implies $\epsilon=\epsilon_{\kappa}$. For $\beta \in L G$ a loop, $\beta^{*} \mathcal{T}_{\kappa}$ is a trivialization of $\beta^{*} \mathscr{R}_{x}^{\nabla}\left(\mathbf{I}_{\epsilon}\right)$, and thus an element of $\mathscr{T}_{\mathscr{R}_{x}^{\nabla}\left(\mathbf{I}_{\epsilon}\right)}^{\nabla}$ over $\beta$. We have

$$
\mathscr{T}_{\mathcal{T}_{\kappa}}^{\nabla}\left(\beta^{*} \mathcal{T}_{\kappa}\right)=\beta^{*} \mathcal{T}_{\kappa} \circ \beta^{*} \mathcal{T}_{\kappa}^{-1}=\mathrm{id}_{\mathcal{I}_{\beta^{*} \rho_{\kappa}}}
$$

considered as an element of $\mathscr{T}_{\mathcal{I}_{\rho_{\kappa}}}^{\nabla}$. Under the canonical trivialization $t_{\rho_{\kappa}}$, this element is equal to $(\beta, 1) \in L X \times \mathrm{U}(1)$.

On the other hand, we compute the element $p:=\varphi_{\mathbf{I}_{\epsilon}}\left(\beta^{*} \mathcal{T}_{\kappa}\right) \in \mathbf{I}_{\epsilon}$ following the definition of $\varphi$ given in Walb, Section 6.2]. We have to consider the space $Z:=S^{1}{ }_{\beta} \times{ }_{\mathrm{ev}_{1}} P_{x} X$ as the subduction of $\beta^{*} \mathscr{R}_{x}^{\nabla}\left(\mathbf{I}_{\epsilon}\right)$ and over $Z$ the bundle $\mathbf{I}_{-\kappa}$, pulled back along the projection $Z \rightarrow P_{x} X$. Over $Z \times_{S^{1}} Z$ the trivialization $\beta^{*} \mathcal{T}_{\kappa}$ has the identity morphism

$$
\text { id }: \cup_{x}^{*} \mathbf{I}_{\epsilon} \otimes \operatorname{pr}_{2}^{*} \mathbf{I}_{-\kappa} \longrightarrow \operatorname{pr}_{1}^{*} \mathbf{I}_{-\kappa},
$$

also pulled back along $Z \times_{S^{1}} Z \rightarrow P_{x} X^{[2]}$. We represent the loop $\beta$ by a path $\gamma \in P_{x} X$ with $\gamma(1)=\beta(0)$ and paths $\gamma_{k} \in P X$ with $\gamma_{k}(0)=\beta(0)$ and $\gamma_{k}(1)=\beta\left(\frac{1}{2}\right)$, related via a thin homotopy $h:\left(\gamma_{1} \star \gamma\right) \cup\left(\gamma_{2} \star \gamma\right) \rightarrow \beta$. In $Z$ we consider the retracting paths $\alpha_{i}$ with $\alpha_{i}(0)=(0$, id $\star \gamma)$ and $\alpha_{i}(1)=\left(\frac{1}{2}, \gamma_{i} \star \gamma\right)$. Then, the prescription is

$$
p=\left(\beta, \exp 2 \pi \mathrm{i}\left(-\int_{\alpha_{2} \star \overline{\alpha_{1}}} \operatorname{pr}^{*} \kappa\right)\right)=\left(\beta, \exp 2 \pi \mathrm{i}\left(\int_{\alpha_{1}} \operatorname{pr}^{*} \kappa-\int_{\alpha_{2}} \operatorname{pr}^{*} \kappa\right)\right) .
$$

Since the paths $\alpha_{i}$ are retractions and the path splitting $\kappa$ is contractible, both integrals vanish separately. Thus, we have $p=(\beta, 1)$; this yields the claimed equality. 


\section{Table of terminology}

Fusion product

- multiplicative $\lambda: \mathcal{L}_{\gamma_{1} \cup \gamma_{2}} \otimes \mathcal{L}_{\gamma_{2} \cup \gamma_{3}} \rightarrow \mathcal{L}_{\gamma_{1} \cup \gamma_{3}}$

Page 4

$\lambda$ is a group homomorphism
Page 4

Page 5

Page 5

Page 5

Page 5

Page 5

Page 4

Page 5

Connection

— thin

- superficial

- compatible

— symmetrizing

- fusive induces a thin homotopy equivariant structure

thin and its holonomy is thin homotopy invariant

fusion product is connection-preserving

induced thin homotopy equivariant structure is symmetrizing

compatible and symmetrizing
Page 13

Page 13

Page 14

Page 14

Page 14

Path splitting of $\epsilon \in \Omega^{k}(L G \times L G)$

$\kappa \in \Omega^{k}(P G \times P G)$ with $\epsilon_{\gamma_{1} \cup \gamma_{2}, \gamma_{1}^{\prime} \cup \gamma_{2}^{\prime}}=\kappa_{\gamma_{1}, \gamma_{1}^{\prime}}-\kappa_{\gamma_{2}, \gamma_{2}^{\prime}}$

Page 14

— multiplicative

$\kappa_{\gamma_{1} \gamma_{1}^{\prime}, \gamma_{2} \gamma_{2}^{\prime}}=\kappa_{\gamma_{1}, \gamma_{2}}+\kappa_{\gamma_{1}^{\prime}, \gamma_{2}^{\prime}}$

Page 14

- contractible

$\int_{\phi_{\gamma}} \kappa=0$, where $\phi_{\gamma}$ is the contraction of a path $\gamma$

Page 14

Thin structure

thin homotopy equivariant structure induced by a superficial Page 14 connection

- fusive

an inducing connection is fusive

Page 14

- multiplicative

the error 1-form of an inducing connection admits a multiplicative and contractible path splitting

- fusive and multiplicative there is an inducing connection with both of above properties Page 15

Central extension of $L G$

— thin fusion

equipped with a multiplicative fusion product and a fusive Page 15 and multiplicative thin structure 


\section{References}

[BM94] J.-L. Brylinski and D. A. McLaughlin, "The geometry of degree four characteristic classes and of line bundles on loop spaces I". Duke Math. J., 75(3):603-638, 1994.

[BM96] J.-L. Brylinski and D. A. McLaughlin, "The Converse of the Segal-Witten Reciprocity Law". Int. Math. Res. Not. IMRN, 8:371-380, 1996.

[Bry] J.-L. Brylinski, "Differentiable cohomology of gauge groups". Preprint. [arxiv:math/0011069]

[Bry93] J.-L. Brylinski, Loop spaces, characteristic classes and geometric quantization. Number 107 in Progr. Math. Birkhäuser, 1993.

[CJM02] A. L. Carey, S. Johnson, and M. K. Murray, "Holonomy on D-branes". J. Geom. Phys., 52(2):186-216, 2002. [arxiv:hep-th/0204199]

$\left[\mathrm{CJM}^{+} 05\right]$ A. L. Carey, S. Johnson, M. K. Murray, D. Stevenson, and B.-L. Wang, "Bundle gerbes for Chern-Simons and Wess-Zumino-Witten theories". Commun. Math. Phys., 259(3):577-613, 2005. [arxiv:math/0410013]

[DK00] J. J. Duistermaat and J. A. C. Kolk, Lie Groups. Springer, 2000.

[Gaw88] K. Gawȩdzki, "Topological actions in two-dimensional quantum field theories". In G. 't Hooft, A. Jaffe, G. Mack, K. Mitter, and R. Stora, editors, Non-perturbative quantum field theory, pages 101-142. Plenum Press, 1988.

[GF93] F. Gabbiani and J. Fröhlich, "Operator algebras and conformal field theory". Commun. Math. Phys., 155(3):569-640, 1993.

[GR02] K. Gawȩdzki and N. Reis, "WZW branes and gerbes". Rev. Math. Phys., 14(12):1281-1334, 2002. [arxiv:hep-th/0205233]

[GW09] K. Gawȩdzki and K. Waldorf, "Polyakov-Wiegmann formula and multiplicative gerbes". J. High Energy Phys., 09(073), 2009. [arxiv:0908.1130]

[Hir76] M. W. Hirsch, Differential Topology. Number 33 in Graduate Texts in Mathematics. Springer, 1976.

[IZ13] P. Iglesias-Zemmour, Diffeology. Number 185 in Mathematical Surveys and Monographs. AMS, 2013.

[Los92] M. V. Losik, "Fréchet Manifolds as Diffeological Spaces". Soviet. Math., 5:36-42, 1992.

[Mei02] E. Meinrenken, "The basic gerbe over a compact simple Lie group". Enseign. Math., II. Sér., 49(3-4):307-333, 2002. [arxiv:math/0209194]

[Mic87] J. Mickelsson, "Kac-Moody groups, topology of the Dirac determinant bundle and fermionization". Commun. Math. Phys., 110:173-183, 1987.

[Mur96] M. K. Murray, "Bundle gerbes". J. Lond. Math. Soc., 54:403-416, 1996. [arxiv:dg-ga/9407015]

[NW13] T. Nikolaus and K. Waldorf, "Lifting problems and transgression for non-abelian gerbes". Adv. Math., 242:50-79, 2013. [arxiv:1112.4702]

[PS86] A. Pressley and G. Segal, Loop groups. Oxford Univ. Press, 1986.

[Seg04] G. Segal, "The definition of conformal field theory". In Topology, geometry and quantum field theory, volume 308 of London Math. Soc. Lecture Note Ser., pages 423-577. Cambridge Univ. Press, 2004.

[Ste00] D. Stevenson, The geometry of bundle gerbes. PhD thesis, University of Adelaide, 2000. [arxiv:math.DG/0004117]

[SW09] U. Schreiber and K. Waldorf, "Parallel transport and functors". J. Homotopy Relat. Struct., 4:187-244, 2009. [arxiv:0705.0452v2] 
[Wala] K. Waldorf, "Spin structures on loop spaces that characterize string manifolds". Algebr. Geom. Topol., to appear. [arxiv:1209.1731]

[Walb] K. Waldorf, "Transgression to loop spaces and its inverse, II: Gerbes and fusion bundles with connection". Asian J. Math., to appear. [arxiv:1004.0031]

[Wal07a] K. Waldorf, Algebraic structures for bundle gerbes and the Wess-Zumino term in conformal field theory. PhD thesis, Universität Hamburg, 2007.

[Wal07b] K. Waldorf, "More morphisms between bundle gerbes". Theory Appl. Categ., 18(9):240-273, 2007. [arxiv:math.CT/0702652]

[Wal10] K. Waldorf, "Multiplicative bundle gerbes with connection". Differential Geom. Appl., 28(3):313-340, 2010. [arxiv:0804.4835v4]

[Wal11] K. Waldorf, "A loop space formulation for geometric lifting problems". J. Aust. Math. Soc., 90:129-144, 2011. [arxiv:1007.5373]

[Wal12a] K. Waldorf, "A construction of string 2-group models using a transgression-regression technique". In C. L. Aldana, M. Braverman, B. Iochum, and C. Neira-Jiménez, editors, Analysis, Geometry and Quantum Field Theory, volume 584 of Contemp. Math., pages 99-115. AMS, 2012. [arxiv:1201.5052]

[Wal12b] K. Waldorf, "Transgression to loop spaces and its inverse, I: Diffeological bundles and fusion maps". Cah. Topol. Géom. Différ. Catég., LIII:162-210, 2012. [arxiv:0911.3212]

[Wal12c] K. Waldorf, "Transgression to loop spaces and its inverse, III: Gerbes and thin fusion bundles". Adv. Math., 231:3445-3472, 2012. [arxiv:1109.0480]

Ernst-Moritz-Arndt-Universität Greifswald

Institut für Mathematik und Informatik

Walther-Rathenau-Str. 47

D-17487 Greifswald

konrad.waldorf@uni-greifswald.de 